\title{
Golden Times for Allenes: Gold-Catalyzed Cycloisomerization of $\beta$-Hydroxyallenes to Dihydropyrans
}

\section{Birgit Gockel and Norbert Krause*}

Organic Chemistry II, Dortmund University, D-44221 Dortmund, Germany

norbert.krause@uni-dortmund.de

\section{Supporting Information}

\section{Typical procedure}

To a solution of the $\beta$-functionalized allene $(0.2 \mathrm{mmol})$ in $3 \mathrm{~mL}$ of dry solvent under argon was added $1-5 \mathrm{~mol} \%$ of the precatalyst. The mixture was stirred at room temperature and the reaction was monitored by TLC. After completion, the mixture was filtered through celite, the solvent was removed in vacuo and the crude product was purified by flash column chromatography on silica gel with cyclohexane/ethyl acetate (10:1).

\section{2-Butyl-2-methyl-5,6-dihydro-2H-pyran (5)}

${ }^{1} \mathrm{H}-\mathrm{NMR}\left(500 \mathrm{MHz}, \mathrm{C}_{6} \mathrm{D}_{6}\right): \delta=5.64-5.61(\mathrm{~m}, 1 \mathrm{H}, 4-\mathrm{H}), 5.45\left(\mathrm{dt},{ }^{3} \mathrm{~J}_{\mathrm{HH}}=10.2 \mathrm{~Hz},{ }^{4} \mathrm{~J}_{\mathrm{HH}}=1.9 \mathrm{~Hz}\right.$, 1 H, 3-H), 3.68-3.60 (m, 2 H, 6-H), 1.87-1.85 (m, 1 H, 5-H), 1.66-1.60 (m, 1 H, 5-H), 1.57-1.36 (m, $4 \mathrm{H}, 1^{\prime}$ - $\mathrm{H}, 2$ ' - $\mathrm{H}$ ), 1.29 (tq, ${ }^{3} \mathrm{~J}_{\mathrm{HH}}=7.3 \mathrm{~Hz},{ }^{3} \mathrm{~J}_{\mathrm{HH}}=7.3 \mathrm{~Hz}, 2 \mathrm{H}, 3$ '-H), 1.20 (s, $3 \mathrm{H}, 2-\mathrm{CH}$ ), 0.90 (t, $\left.{ }^{3} \mathrm{~J}_{\mathrm{HH}}=7.3 \mathrm{~Hz}, 3 \mathrm{H}, 4{ }^{\prime}-\mathrm{H}\right) .{ }^{13} \mathrm{C}-\mathrm{NMR}\left(125 \mathrm{MHz}, \mathrm{C}_{6} \mathrm{D}_{6}\right): \delta=135.0(+, \mathrm{C}-3), 123.6(+, \mathrm{C}-4), 73.5(\times$, C-2), 59.1 (-, C-6), 41.1 (-, C-1'), 26.2 (-, C-5/C-2'/C-3’), 25.4 (-, C-5/C-2'/C-3’), 25.2 (+, 2-CH3), 23.6 (-, C-5/C-2'/C-3'), 14.4 (+, C-4’).

\section{2-t-Butyl-2-methyl-5,6-dihydro-2H-pyran (8a)}

${ }^{1} \mathrm{H}-\mathrm{NMR}\left(400 \mathrm{MHz}, \mathrm{C}_{6} \mathrm{D}_{6}\right.$ ): $\delta=$ 5.67-5.58 (m, 2 H, 3-H, 4-H), 3.69-3.64 (m, $\left.1 \mathrm{H}, 6-\mathrm{H}\right), 1.58-1.52$ (m, 1 H, 6-H), 2.07-1.98 (m, 1 H, 5-H), 1.43-1.37 (m, 1 H, 5-H), 1.14 (s, 3 H, 2-CH3), 1.04 (s, 9 H, 2-C( $\left.\left(\mathrm{CH}_{3}\right)_{3}\right) .{ }^{13} \mathrm{C}-\mathrm{NMR}\left(100 \mathrm{MHz}, \mathrm{C}_{6} \mathrm{D}_{6}\right): \delta=132.7$ (+, C-3), 124.5 (+, C-4), 77.7 (×, C-2), 59.3 (-, C-6), $37.9\left(\times, 2-\underline{C}\left(\mathrm{CH}_{3}\right)_{3}\right), 25.4\left(+, 2-\mathrm{C}\left(\underline{\mathrm{CH}_{3}}\right)_{3}\right), 25.3(-, \mathrm{C}-5), 19.0\left(+, 2-\mathrm{CH}_{3}\right)$. 


\section{2-t-Butyl-2-butyl-5,6-dihydro-2H-pyran (8b)}

${ }^{1} \mathrm{H}-\mathrm{NMR}\left(400 \mathrm{MHz}, \mathrm{C}_{6} \mathrm{D}_{6}\right): \delta=5.84-5.80$ (m, $\left.1 \mathrm{H}, 4-\mathrm{H}\right)$ ) 5.55-5.53 (m, $\left.1 \mathrm{H}, 3-\mathrm{H}\right)$, 3.75-3.66 (m, 2 H, 6-H), 1.88-1.75 (m, 1 H, 5-H), 1.63-1.46 (m, 3 H, 5-H, 1'-H), 1.38-1.20 (m, 4 H, 2'-H, 3'-H), 1.05 (s, $\left.9 \mathrm{H}, 2-\mathrm{C}\left(\mathrm{CH}_{3}\right)_{3}\right), 0.92\left(\mathrm{t},{ }^{3} \mathrm{~J}_{\mathrm{HH}}=7.3 \mathrm{~Hz}, 3 \mathrm{H}, 4{ }^{\prime}-\mathrm{H}\right) .{ }^{13} \mathrm{C}-\mathrm{NMR}\left(100 \mathrm{MHz}, \mathrm{C}_{6} \mathrm{D}_{6}\right): \delta=131.3$ (+, C-3), 125.8 (+, C-4), 79.4 (×, C-2), 61.7 (-, C-6), 39.5 (×, 2- $\left.\underline{\mathrm{C}}\left(\mathrm{CH}_{3}\right)_{3}\right), 35.9$ (-, C-1'), 28.2 (-, C5/C-2'/C-3'), 26.2 (+, 2-C( $\left.\left.\underline{\mathrm{CH}}_{3}\right)_{3}\right), 25.0$ (-, C-5/C-2'/C-3'), 24.1 (-, C-5/C-2'/C-3'), 14.5 (+, C-4').

\section{6-Butyl-2,2,6-trimethyl-3,6-dihydro-2H-pyran (8c)}

${ }^{1} \mathrm{H}-\mathrm{NMR}\left(400 \mathrm{MHz}, \mathrm{C}_{6} \mathrm{D}_{6}\right): \delta=5.66-5.61(\mathrm{~m}, 1 \mathrm{H}, 4-\mathrm{H}), 5.48\left(\mathrm{dd},{ }^{3} \mathrm{~J}_{\mathrm{HH}}=10.3 \mathrm{~Hz},{ }^{4} \mathrm{~J}_{\mathrm{HH}}=1.3 \mathrm{~Hz}\right.$, 1 H, 3-H), 1.90-1.85 (m, 1 H, 5-H), 1.69-1.64 (m, 1 H, 5-H), 1.61-1.29 (m, 6 H, 1'-H, 2'-H, 3'-H), 1.26 (s, $\left.3 \mathrm{H}, 2-\mathrm{CH}_{3}\right), 1.24$ (s, $\left.3 \mathrm{H}, 6-\mathrm{CH}_{3}\right), 1.21$ (s, $\left.3 \mathrm{H}, 6-\mathrm{CH}_{3}\right), 0.92\left(\mathrm{t},{ }^{3} \mathrm{~J}_{\mathrm{HH}}=7.3 \mathrm{~Hz}, 3 \mathrm{H}, 4^{\prime}-\mathrm{H}\right)$. ${ }^{13} \mathrm{C}-\mathrm{NMR}\left(100 \mathrm{MHz}, \mathrm{C}_{6} \mathrm{D}_{6}\right): \delta=133.43(+, \mathrm{C}-3), 121.9(+, \mathrm{C}-4), 73.7(\times, \mathrm{C}-2), 70.0(\times, \mathrm{C}-6), 43.6$ (-, C-1'), 36.1 (-, C-5), 30.9 (+, 2-CH $\left.\mathrm{CH}_{3}\right), 28.6$ (-, 6- $\left.\mathrm{CH}_{3}\right), 28.2$ (+, 6-CH), 26.3 (-, C-2'/C-3’), 23.5 (-, C-2'/C-3'), 14.5 (+, C-4').

\section{Ethyl 6-butyl-2,2,6-trimethyl-3,6-dihydro-2H-pyran-3-carboxylate (8d)}

${ }^{1} \mathrm{H}-\mathrm{NMR}\left(400 \mathrm{MHz}, \mathrm{C}_{6} \mathrm{D}_{6}\right): \delta=5.94 \star / 5.78\left(2 \mathrm{dd},{ }^{3} \mathrm{~J}_{\mathrm{HH}}=3.8 \mathrm{~Hz} / 10.3 \mathrm{~Hz}, 1 \mathrm{H}, 4-\mathrm{H}\right), 5.64 / 5.46 *$ (2dd, $\left.{ }^{3} \mathrm{~J}_{\mathrm{HH}}=2.8 \mathrm{~Hz} / 10.3 \mathrm{~Hz}, 1 \mathrm{H}, 4-\mathrm{H}\right), 3.98-3.84$ (m, $2 \mathrm{H}, \mathrm{OC}_{2} \mathrm{CH}_{3}$ ), 3.08-3.07*/3.01-2.99 (2m, 1 H, 5-H), 1.52*/1.41 (2s, 3 H, 6-CH $), 1.33-1.24$ (m, 6 H, 1’-H, 2’-H, 3’-H), 1.21*/1.18 (2s, 3 H,

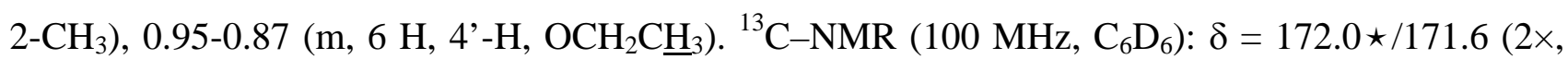
$\left.\mathrm{O} \underline{\mathrm{C}}(\mathrm{O}) \mathrm{CH}_{3}\right), 135.0 / 133.3 *$ (2+, C-3), $121.6 * / 120.0$ (2+, C-4), 74.0*/73.7 (2×, C-6), $72.5 * / 72.2$ (2×, C-2), 60.4*/ 60.3 (2-, $\left.\mathrm{OCL}_{2} \mathrm{CH}_{3}\right), 50.4 * / 50.3$ (2+, C-5), 43.7*/42.0 (2-, C-1'), 30.7/30.6* (2+, 2$\left.\mathrm{CH}_{3}\right), 27.7 * / 27.4$ (2+, 6- $\left.\mathrm{CH}_{3}\right), 26.4 / 26.2 *$ (2-, C-2'), 26.0/24.9* (2+, 6- $\left.\mathrm{CH}_{3}\right), 23.7 / 23.4 *$ (2-, C-3’), $14.5 * / 14.4\left(2+, \mathrm{C}-4{ }^{\prime}\right), 14.3 / 14.2 *\left(2+, \mathrm{OCH}_{2} \mathrm{CH}_{3}\right)$.

\section{1-Hydroxy-3,6,6-trimethylhepta-3,4-dien-2-yl acetate (9a)}

${ }^{1} \mathrm{H}-\mathrm{NMR}\left(400 \mathrm{MHz}, \mathrm{C}_{6} \mathrm{D}_{6}\right.$ ): $\delta=5.41$ (m, $\left.1 \mathrm{H}, 2-\mathrm{H}\right), 5.15$ (m, $\left.1 \mathrm{H}, 5-\mathrm{H}\right), 3.70-3.69$ (m, $\left.2 \mathrm{H}, 1-\mathrm{H}\right)$, $1.79\left(\mathrm{~m}, 3 \mathrm{H}, \mathrm{OC}(\mathrm{O}) \mathrm{CH}_{3}\right), 1.66\left(\mathrm{~d},{ }^{5} \mathrm{~J}_{\mathrm{HH}}=2.3 \mathrm{~Hz}, 3 \mathrm{H}, 3-\mathrm{CH}_{3}\right), 0.98\left(\mathrm{~s}, 9 \mathrm{H}, \mathrm{C}\left(\mathrm{CH}_{3}\right)_{3}\right)$.

${ }^{13} \mathrm{C}-\mathrm{NMR}\left(100 \mathrm{MHz}, \mathrm{C}_{6} \mathrm{D}_{6}\right): \delta=199.4(\times, \mathrm{C}-4), 170.5\left(\times, \mathrm{OC}(\mathrm{O}) \mathrm{CH}_{3}\right), 105.2(+, \mathrm{C}-5), 99.1(\times$, C-3), 75.7 (+, C-2), 63.7 (-, C-1), 32.2 (×, C-6), 30.2 (+, C( $\left.\left.\mathrm{CH}_{3}\right)_{3}\right), 20.8\left(+, \mathrm{OC}(\mathrm{O}) \mathrm{CH}_{3}\right), 16.5(+$, 3- $\left.\mathrm{CH}_{3}\right)$. 


\section{Cis-6-t-Butyl-4-methyl-3,6-dihydro- $2 H$-pyran-3-yl acetate (10a)}

${ }^{1} \mathrm{H}-\mathrm{NMR}\left(400 \mathrm{MHz}, \mathrm{C}_{6} \mathrm{D}_{6}\right.$ ): $\delta=5.56$ (m, $\left.1 \mathrm{H}, 3-\mathrm{H}\right), 4.91$ (m, $\left.1 \mathrm{H}, 5-\mathrm{H}\right), 4.11-4.08$ (m, $\left.1 \mathrm{H}, 6-\mathrm{H}\right)$, 3.39-3.35 (m, 2 H, 2-H, 6-H), 1.66 (s, 3 H, OC(O)CH $)_{3}$ ), 1.61 (s, 3 H, 4- $\left.\mathrm{CH}_{3}\right), 0.96$ (s, 9 H, C( $\left.\left(\mathrm{CH}_{3}\right)_{3}\right)$. ${ }^{13} \mathrm{C}-\mathrm{NMR}\left(100 \mathrm{MHz}, \mathrm{C}_{6} \mathrm{D}_{6}\right): \delta=170.5\left(\times, \mathrm{OC}(\mathrm{O}) \mathrm{CH}_{3}\right), 131.1(\times, \mathrm{C}-4), 128.0(+, \mathrm{C}-3), 82.0(+$, C-1), 68.4 (-, C-6), 68.4 (+, C-5), 34.6 (×, $\left.\mathrm{C}\left(\mathrm{CH}_{3}\right)_{3}\right), 26.0\left(+, \mathrm{C}\left(\mathrm{CH}_{3}\right)_{3}\right), 20.7\left(+, 4-\mathrm{CH}_{3} / \mathrm{OC}(\mathrm{O}) \underline{\mathrm{C}} \mathrm{H}_{3}\right)$, $20.7\left(+, 4-\mathrm{CH}_{3} / \mathrm{OC}(\mathrm{O}) \underline{\mathrm{CH}}_{3}\right)$.

\section{Trans-2-Acetoxymethyl-5-t-butyl-3,5-dimethyl-2,5-dihydrofuran (11a)}

${ }^{1} \mathrm{H}-\mathrm{NMR}\left(400 \mathrm{MHz}, \mathrm{C}_{6} \mathrm{D}_{6}\right): \delta=5.23$ (m 1, H- 4-H), 4.62-4.61 (m, $\left.1 \mathrm{H}, 2-\mathrm{H}\right), 4.59-4.44$ (m, $1 \mathrm{H}$,

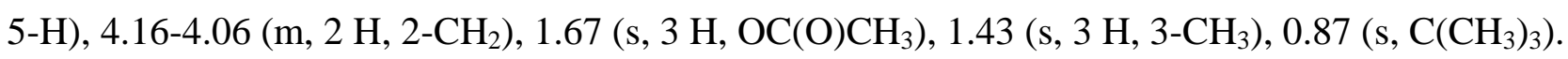

${ }^{13} \mathrm{C}-\mathrm{NMR}\left(100 \mathrm{MHz}, \mathrm{C}_{6} \mathrm{D}_{6}\right): \delta=170.1\left(\times, \mathrm{O} \underline{\mathrm{C}}(\mathrm{O}) \mathrm{CH}_{3}\right), 136.6(\times, \mathrm{C}-3), 124.3(+, \mathrm{C}-4), 94.1(+$, C-5), 86.3 (+, C-2), 65.5 (-, 2- $\left.\mathrm{CH}_{2}\right), 35.7\left(\times, \underline{\mathrm{C}}\left(\mathrm{CH}_{3}\right)_{3}\right), 25.7\left(+, \mathrm{C}(\underline{\mathrm{CH}})_{3}\right), 20.4\left(+, \mathrm{OC}(\mathrm{O}) \underline{\left.\mathrm{CH}_{3}\right),} 12.3\right.$ $\left(+, 3-\mathrm{CH}_{3}\right)$.

\section{Trans-6-t-Butyl-4-methyl-3,6-dihydro-2H-pyran-3-yl acetate (10b)}

${ }^{1} \mathrm{H}-\mathrm{NMR}\left(400 \mathrm{MHz}, \mathrm{C}_{6} \mathrm{D}_{6}\right): \delta=5.55(\mathrm{~m}, 1 \mathrm{H}, 5-\mathrm{H}), 5.50(\mathrm{~m}, 1 \mathrm{H}, 3-\mathrm{H}), 4.25\left(\mathrm{dd},{ }^{2} \mathrm{~J}_{\mathrm{HH}}=10.4 \mathrm{~Hz}\right.$, $\left.{ }^{3} \mathrm{~J}_{\mathrm{HH}}=5.9 \mathrm{~Hz}, 1 \mathrm{H}, 6-\mathrm{H}\right), 3.58(\mathrm{~m}, 1 \mathrm{H}, 2-\mathrm{H}), 3.33\left(\mathrm{dd},{ }^{2} \mathrm{~J}_{\mathrm{HH}}=10.4 \mathrm{~Hz},{ }^{3} \mathrm{~J}_{\mathrm{HH}}=9.1 \mathrm{~Hz}, 1 \mathrm{H}, 6-\mathrm{H}\right)$, 1.62 (s, $\left.3 \mathrm{H}, \mathrm{OC}(\mathrm{O}) \mathrm{CH}_{3}\right), 1.59$ (s, $\left.3 \mathrm{H}, 4-\mathrm{CH}_{3}\right), 0.92$ (s, $\left.9 \mathrm{H}, \mathrm{C}\left(\mathrm{CH}_{3}\right)_{3}\right)$.

${ }^{13} \mathrm{C}-\mathrm{NMR}\left(100 \mathrm{MHz}, \mathrm{C}_{6} \mathrm{D}_{6}\right): \delta=169.8\left(\times, \mathrm{OC}(\mathrm{O}) \mathrm{CH}_{3}\right), 133.9(\times, \mathrm{C}-4), 125.8(+, \mathrm{C}-3), 82.4(+$, C-2), 67.7 (+, C-5), 66.7 (-, C-6), $35.0\left(\times, \underline{\mathrm{C}}\left(\mathrm{CH}_{3}\right)_{3}\right), 25.8$ (+, $\left.\left.\mathrm{C}\left(\underline{\mathrm{CH}_{3}}\right)_{3}\right), 20.3\left(+, 4-\mathrm{CH}_{3} / \mathrm{OC}(\mathrm{O}) \underline{\mathrm{CH}}\right)_{3}\right)$, $18.4\left(+, 4-\mathrm{CH}_{3} / \mathrm{OC}(\mathrm{O}) \underline{\mathrm{CH}}_{3}\right)$.

\section{Cis-2-Acetoxymethyl-5-t-butyl-3,5-dimethyl-2,5-dihydrofuran (11b)}

${ }^{1} \mathrm{H}-\mathrm{NMR}\left(400 \mathrm{MHz}, \mathrm{C}_{6} \mathrm{D}_{6}\right): \delta=5.18(\mathrm{~m}, 1 \mathrm{H}, 4-\mathrm{H}), 4.63-4.62(\mathrm{~m}, 1 \mathrm{H}, 2-\mathrm{H}), 4.39-4.37$ (m, $1 \mathrm{H}$, 5-H), $4.23\left(\mathrm{dd},{ }^{2} \mathrm{~J}_{\mathrm{HH}}=11.8 \mathrm{~Hz},{ }^{3} \mathrm{~J}_{\mathrm{HH}}=3.3 \mathrm{~Hz}, 1 \mathrm{H}, 2-\mathrm{CH}_{2}\right), 4.14\left(\mathrm{dd},{ }^{2} \mathrm{~J}_{\mathrm{HH}}=11.8 \mathrm{~Hz},{ }^{3} \mathrm{~J}_{\mathrm{HH}}=5.5 \mathrm{~Hz}\right.$, $\left.1 \mathrm{H}, 2-\mathrm{CH}_{2}\right), 1.66$ (s, $\left.3 \mathrm{H}, \mathrm{OC}(\mathrm{O}) \mathrm{CH}_{3}\right), 1.40$ (s, $\left.3 \mathrm{H}, 3-\mathrm{CH}_{3}\right), 0.91$ (s, $\left.\mathrm{C}\left(\mathrm{CH}_{3}\right)_{3}\right)$.

${ }^{13} \mathrm{C}-\mathrm{NMR}\left(100 \mathrm{MHz}, \mathrm{C}_{6} \mathrm{D}_{6}\right): \delta=170.0\left(\times, \mathrm{OC}(\mathrm{O}) \mathrm{CH}_{3}\right), 136.8(\times, \mathrm{C}-3), 124.4(+, \mathrm{C}-4), 93.9(+$, C-5), 85.4 (+, C-2), 65.8 (-, 2- $\left.\mathrm{CH}_{2}\right), 34.5\left(\times, \underline{\mathrm{C}}\left(\mathrm{CH}_{3}\right)_{3}\right), 26.0\left(+, \mathrm{C}(\underline{\mathrm{CH}})_{3}\right), 20.4\left(+, \mathrm{OC}(\mathrm{O}) \underline{\left.\mathrm{CH}_{3}\right),} 12.3\right.$ $\left(+, 3-\mathrm{CH}_{3}\right)$.

\section{Trans-6-Butyl-4-methyl-3,6-dihydro-2H-pyran-3-yl acetate (10c)}

${ }^{1} \mathrm{H}-\mathrm{NMR}\left(400 \mathrm{MHz}, \mathrm{C}_{6} \mathrm{D}_{6}\right): \delta=5.39(\mathrm{~m}, 1 \mathrm{H}, 5-\mathrm{H}), 5.31(\mathrm{~m}, 1 \mathrm{H}, 3-\mathrm{H}), 4.08\left(\mathrm{dd},{ }^{2} \mathrm{~J}_{\mathrm{HH}}=11.3 \mathrm{~Hz}\right.$, $\left.{ }^{3} \mathrm{~J}_{\mathrm{HH}}=5.0 \mathrm{~Hz}, 1 \mathrm{H}, 6-\mathrm{H}\right), 3.95-3.93(\mathrm{~m}, 1 \mathrm{H}, 2-\mathrm{H}), 3.51\left(\mathrm{dd},{ }^{2} \mathrm{~J}_{\mathrm{HH}}=11.3 \mathrm{~Hz},{ }^{3} \mathrm{~J}_{\mathrm{HH}}=6.7 \mathrm{~Hz}, 1 \mathrm{H}\right.$, 
6-H), 1.64 (s, 3 H, 4- $\mathrm{CH}_{3}$ ), 1.59 (s, $3 \mathrm{H}, \mathrm{OC}(\mathrm{O}) \mathrm{CH}_{3}$ ), 1.50-1.37 (m, $\left.2 \mathrm{H}, 1^{\prime}-\mathrm{H}\right), 1.34-1.19$ (m, 4 H, 2'-H, 3'-H), 0.86 (t, $\left.{ }^{3} \mathrm{~J}_{\mathrm{HH}}=7.2 \mathrm{~Hz}, 3 \mathrm{H}, 4^{\prime}-\mathrm{H}\right)$.

${ }^{13} \mathrm{C}-\mathrm{NMR}\left(100 \mathrm{MHz}, \mathrm{C}_{6} \mathrm{D}_{6}\right): \delta=170.0\left(\times, \mathrm{OC}(\mathrm{O}) \mathrm{CH}_{3}\right), 131.2(\times, \mathrm{C}-4), 129.7(+, \mathrm{C}-3), 74.0(+$, C-2), 67.9 (+, C-5), 65.4 (-, C-6), 34.4 (-, C-1’), 27.8 (-, C-2’), 23.0 (-, C-3’), 20.4 (+, 4- $\left.\mathrm{CH}_{3} / \mathrm{OC}(\mathrm{O}) \underline{\mathrm{CH}}_{3}\right), 19.0\left(+, 4-\mathrm{CH}_{3} / \mathrm{OC}(\mathrm{O}) \underline{\mathrm{CH}}_{3}\right), 14.2$ (+, C-4').

\section{Trans-2-Butyl-5-methoxy-5,6-dihydro-2H-pyran (10d)}

${ }^{1} \mathrm{H}-\mathrm{NMR}\left(400 \mathrm{MHz}, \mathrm{C}_{6} \mathrm{D}_{6}\right): \delta=5.29(\mathrm{~m}, 1 \mathrm{H}, 3-\mathrm{H}), 4.25\left(\mathrm{dd},{ }^{2} \mathrm{~J}_{\mathrm{HH}}=10.7 \mathrm{~Hz},{ }^{3} \mathrm{~J}_{\mathrm{HH}}=4.9 \mathrm{~Hz}, 1 \mathrm{H}\right.$, 6-H), 3.97 (m, $1 \mathrm{H}, 2-\mathrm{H}), 3.59$ (m, $1 \mathrm{H}, 5-\mathrm{H}), 3.56$ (dd, $\left.{ }^{2} \mathrm{~J}_{\mathrm{HH}}=10.7 \mathrm{~Hz},{ }^{3} \mathrm{~J}_{\mathrm{HH}}=7.7 \mathrm{~Hz}, 1 \mathrm{H}, 6-\mathrm{H}\right)$, 3.07 (s, $\left.3 \mathrm{H}, \mathrm{OCH}_{3}\right), 1.77$ (s, $\left.3 \mathrm{H}, 4-\mathrm{CH}_{3}\right), 1.63-1.28$ (m, $6 \mathrm{H}, 1^{\prime}-\mathrm{H}, 2^{\prime}-\mathrm{H}, 3$ ' $\left.-\mathrm{H}\right), 0.88$ (t, ${ }^{3} \mathrm{~J}_{\mathrm{HH}}=$ $\left.7.3 \mathrm{~Hz}, 3 \mathrm{H}, 4^{\prime}-\mathrm{H}\right)$.

${ }^{13} \mathrm{C}-\mathrm{NMR}\left(100 \mathrm{MHz}, \mathrm{C}_{6} \mathrm{D}_{6}\right): \delta=134.2(\times, \mathrm{C}-4), 127.9$ (+, C-3), 74.8 (+, C-2/C-5), $74.4(+, \mathrm{C}-2 /$ C-5), 66.4 (-, C-6), 57.0 (+, $\mathrm{OCH}_{3}$ ), 35.2 (-, C-1'), 27.8 (-, C-2’), 23.1 (-, C-3’), 19.1 (+, 4-CH 3 ), $14.3(+, \mathrm{C}-4$ ').

\section{Trans-4-Methyl-6-phenyl-3,6-dihydro-2H-pyran-3-yl acetate (10e)}

${ }^{1} \mathrm{H}-\mathrm{NMR}\left(400 \mathrm{MHz}, \mathrm{C}_{6} \mathrm{D}_{6}\right.$ ): $\delta=7.40-7.38 / 7.27-7.25^{*}$ (2m, $2 \mathrm{H}$, Aryl-H), 7.18/ 7.18-7.17* (2m, $2 \mathrm{H}$, Aryl-H), 7.12-7.11/7.10-7.09 (2m, 1 H, Aryl-H), 5.52*/5.45 (2m, 1 H, 3-H), 5.30-5.28*/5.02 (2m, $1 \mathrm{H}, 5-\mathrm{H}), 5.04 * / 4.74(2 \mathrm{~m}, 1 \mathrm{H}, 2-\mathrm{H}), 4.13 / 3.95^{*}\left(2 \mathrm{dd},{ }^{2} \mathrm{~J}_{\mathrm{HH}}=11.9 \mathrm{~Hz},{ }^{3} \mathrm{~J}_{\mathrm{HH}}=4.2 \mathrm{~Hz}, 1 \mathrm{H}, 6-\mathrm{H}\right)$, $3.64 * / 3.53\left(2 \mathrm{dd},{ }^{2} \mathrm{~J}_{\mathrm{HH}}=11.9 \mathrm{~Hz},{ }^{3} \mathrm{~J}_{\mathrm{HH}}=5.1 \mathrm{~Hz}, 1 \mathrm{H}, 6-\mathrm{H}\right), 1.67 * / 1.66\left(2 \mathrm{~s}, 3 \mathrm{H}, \mathrm{OC}(\mathrm{O}) \mathrm{CH}_{3}\right)$, $1.60 * / 1.56\left(2 \mathrm{~s}, 3 \mathrm{H}, 4-\mathrm{CH}_{3}\right)$.

${ }^{13} \mathrm{C}-\mathrm{NMR}\left(100 \mathrm{MHz}, \mathrm{C}_{6} \mathrm{D}_{6}\right): \delta=170.5 / 170.1^{*}\left(2 \times, \mathrm{OC}(\mathrm{O}) \mathrm{CH}_{3}\right), 141.5 / 140.7^{*}\left(2 \times, \mathrm{C}-1^{\prime}\right), 131.7^{*}(\times$, C-4), 130.7*/129.7 (C-4’), 128.6*/128.5 (2+, C-3’, C-5’), 128.0/ 127.9* (2+, C-2’, C-6’), 127.4* (+, C-3), 76.8/75.4* (2+, C-5), 68.0/65.0* (2-, C-6), 67.9/67.5* (2+, C-2), 20.6/20.4* (2+, OC(O)ㅡㅡㄴ ${ }_{3}$ ), $20.2 / 19.3 *\left(2+, 4-\mathrm{CH}_{3}\right)$.

\section{Trans-5-Methoxy-4-methyl-2-phenyl-5,6-dihydro-2H-pyran (10f)}

${ }^{1} \mathrm{H}-\mathrm{NMR}\left(400 \mathrm{MHz}, \mathrm{C}_{6} \mathrm{D}_{6}\right): \delta=7.45-7.40 / 7.33-7.32 *(2 \mathrm{~m}, 2 \mathrm{H}$, Aryl-H), 7.23-7.08 (m, $3 \mathrm{H}$, Aryl-H), 5.71/5.47* (2m, $1 \mathrm{H}, 3-\mathrm{H}), 5.26 / 5.02 *(2 \mathrm{~m}, 1 \mathrm{H}, 2-\mathrm{H}), 4.11 / 3.95^{*}\left(2 \mathrm{dd},{ }^{2} \mathrm{~J}_{\mathrm{HH}}=11.4 \mathrm{~Hz}\right.$, 
$\left.{ }^{3} \mathrm{~J}_{\mathrm{HH}}=4.6 \mathrm{~Hz}, 1 \mathrm{H}, 6-\mathrm{H}\right), 3.49-3.47 * / 3.45-3.43(2 \mathrm{~m}, 1 \mathrm{H}, 5-\mathrm{H}), 3.11 * / 3.10\left(2 \mathrm{~s}, 3 \mathrm{H}, \mathrm{OCH}_{3}\right)$, $1.74 * / 1.68\left(2 \mathrm{~s}, 3 \mathrm{H}, 4-\mathrm{CH}_{3}\right)$.

${ }^{13} \mathrm{C}-\mathrm{NMR}\left(100 \mathrm{MHz}, \mathrm{C}_{6} \mathrm{D}_{6}\right): \delta=142.0 / 141.7^{*}\left(\times, \mathrm{C}^{\prime} \mathbf{1}^{\prime}\right), 137.4 / 134.1^{*}(\mathrm{C}-4), 131.1$ (×, C-4’), 128.6/128.5* (+, C-3’, C-5’), 127.8/127.7* (+, C-2’, C-6’), 127.2/127.1* (+, C-3), 77.0/76.0* ${ }^{*}$, C-5), $74.2 * / 73.9(+, \mathrm{C}-2), 66.9 / 65.7^{*}(-, \mathrm{C}-6), 56.6 * / 55.4\left(+, \mathrm{OCH}_{3}\right), 20.5 / 19.3 *\left(+, 4-\mathrm{CH}_{3}\right)$.

\section{2-Butyl-2-methyl-1,2,5,6-tetrahydropyridin (13)}

${ }^{1} \mathrm{H}-\mathrm{NMR}\left(400 \mathrm{MHz}, \mathrm{C}_{6} \mathrm{D}_{6}\right.$ ): $\delta$ = 5.59-5.56 (m, $\left.1 \mathrm{H}, 4-\mathrm{H}\right)$, 5.43-5.40 (m, $\left.1 \mathrm{H}, 3-\mathrm{H}\right), 2.84-2.82$ (m, 2 H, 6-H), 1.91-1.86 (m, 1 H, 5-H), 1.78-1.72 (m, 1 H, 5-H), 1.50-1.21 (m, 6 H, 1'-H, 2'-H, 3’-H), 1.11 (s, $\left.3 \mathrm{H}, 2-\mathrm{CH}_{3}\right), 0.90$ (t, $\left.{ }^{3} \mathrm{~J}_{\mathrm{HH}}=7.0 \mathrm{~Hz}, 3 \mathrm{H}, 4^{\prime}-\mathrm{H}\right)$.

${ }^{13} \mathrm{C}-\mathrm{NMR}\left(100 \mathrm{MHz}, \mathrm{C}_{6} \mathrm{D}_{6}\right): \delta=135.3$ (+, C-3), 124.3 (+, C-4), 53.4 (×, C-2), 41.7 (-, C-6), 38.6 (-, C-1'), 26.3 (-, C-5), 26.2 (+, 2-CH3), 25.3 (-, C-2’/C-3’), 23.7 (-, C-2’/C-3’), 14.4 (+, C-4’). 


\section{2-Butyl-2-methyl-5,6-dihydro-2H-pyran (5)}

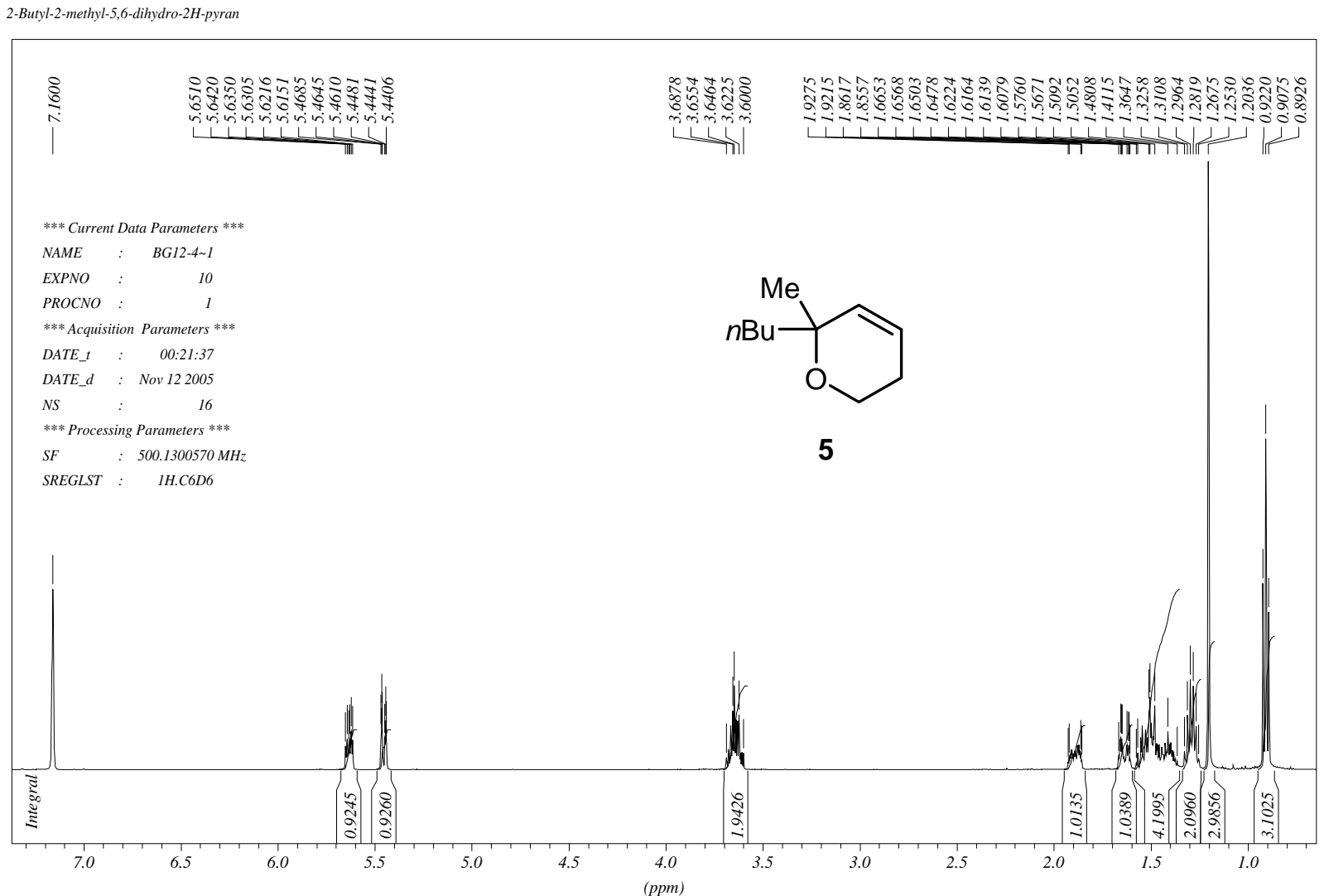

2-Butyl-2-methyl-5,6-dihydro-2H-pyran

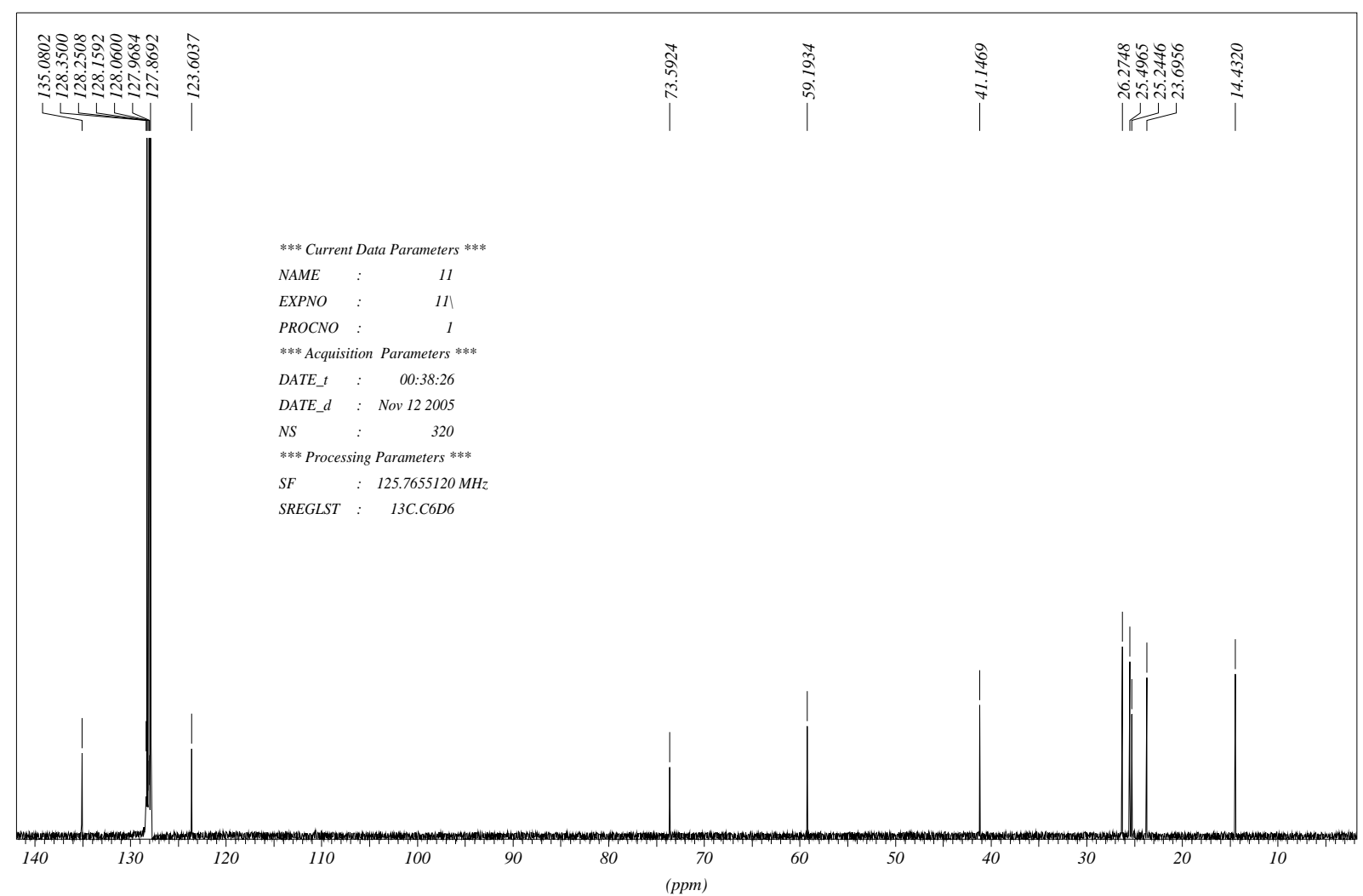


2-t-Butyl-2-methyl-5,6-dihydro-2H-pyran (8a)

2-t-Butyl-2-methyl-5,6-dihydro-2H-pyra

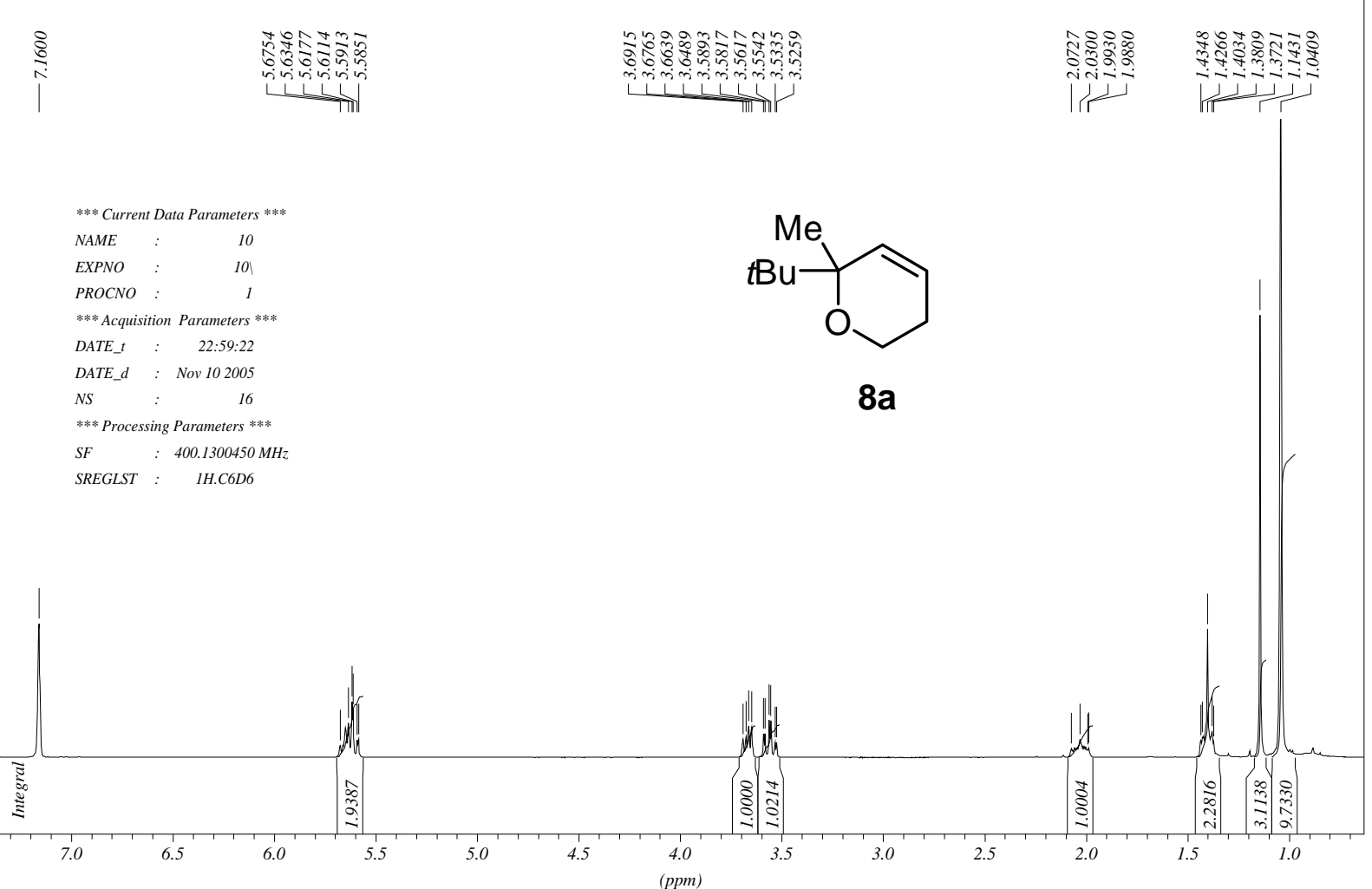

2-t-Butyl-2-methyl-5,6-dihydro-2H-pyran

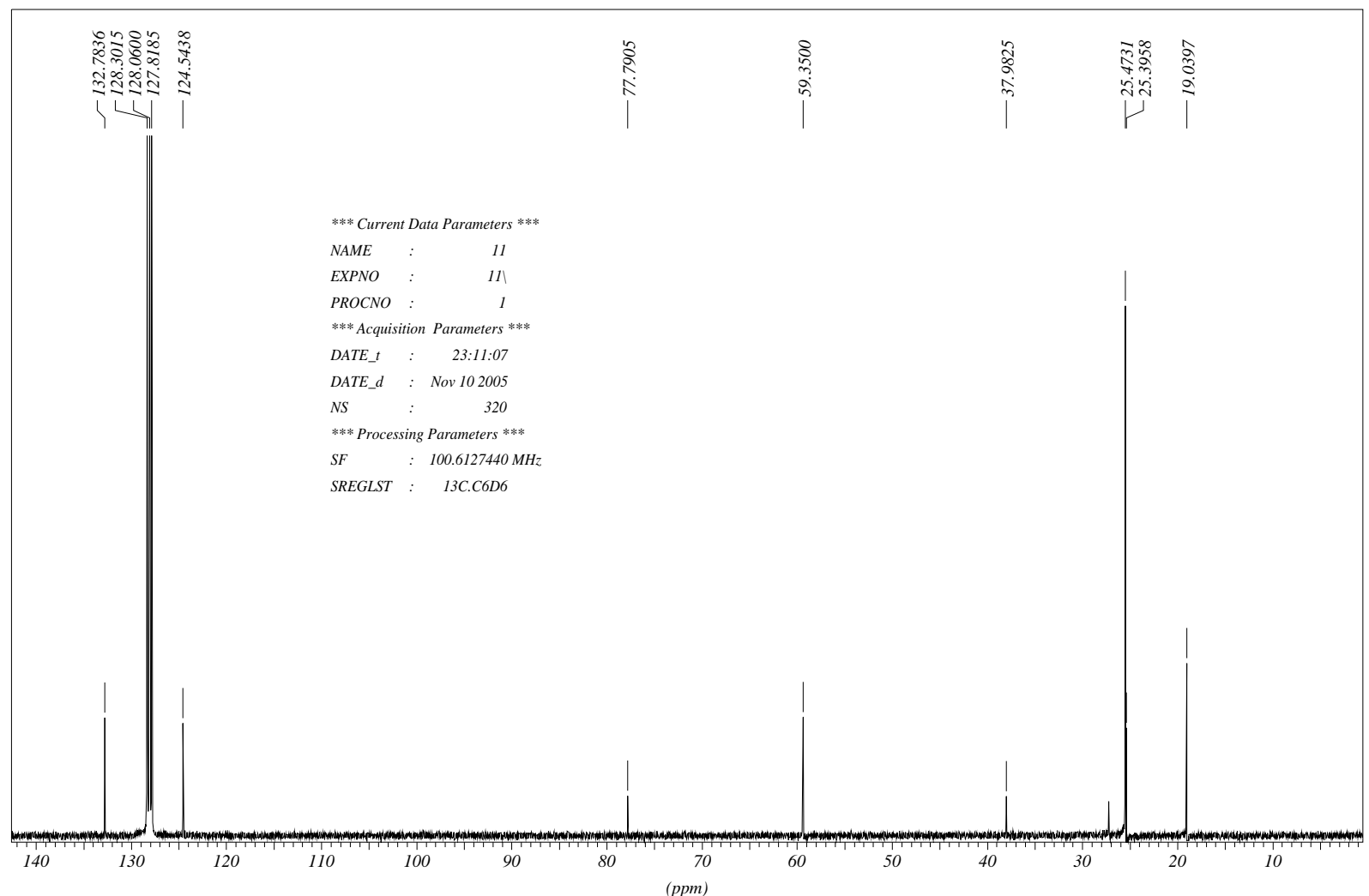


2-t-Butyl-2-butyl-5,6-dihydro-2H-pyran (8b)

2-tert-Butyl-2-butyl-5,6-dihydro-2H-pyran

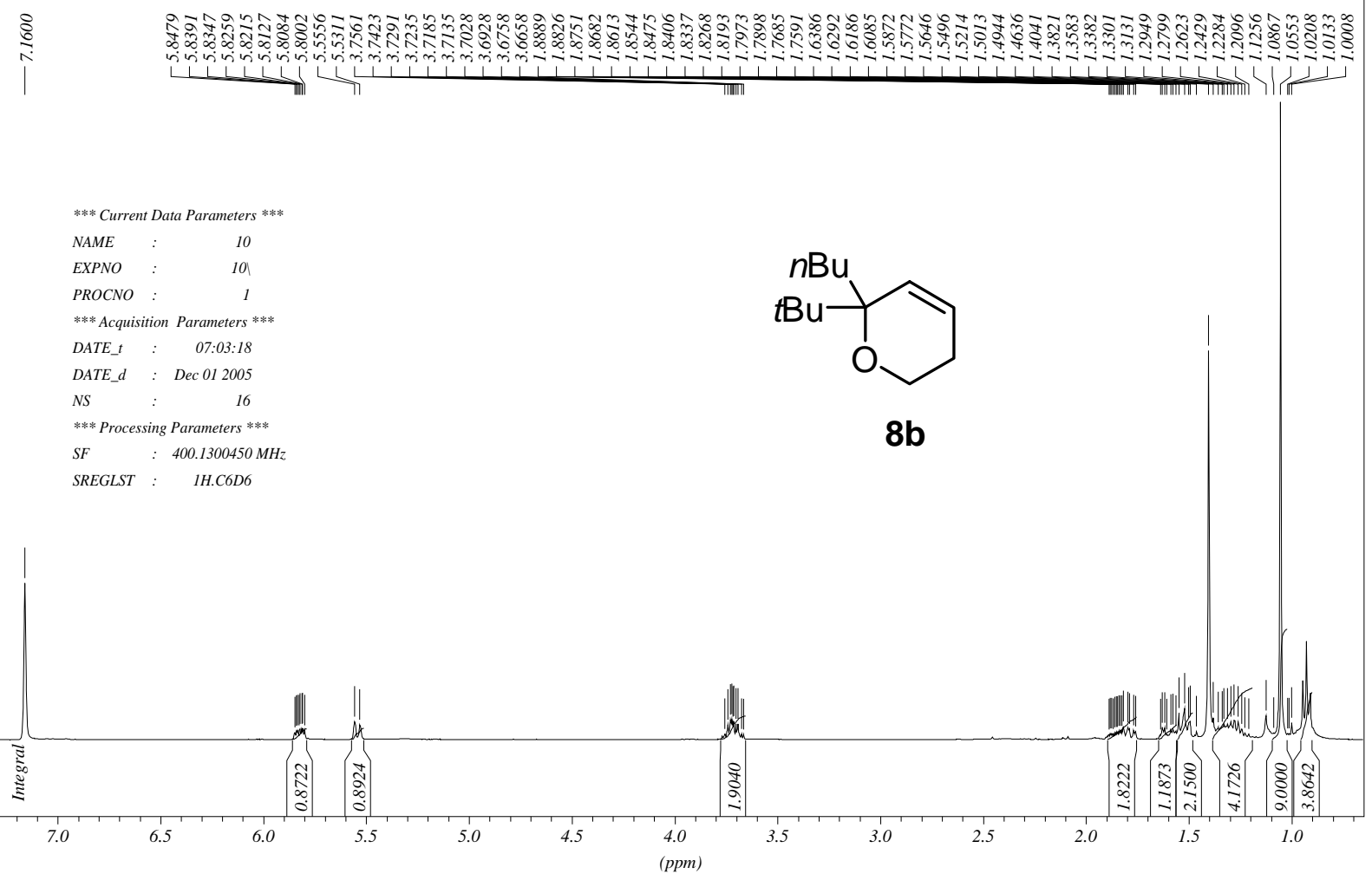

2-tert-Butyl-2-butyl-5,6-dihydro-2H-pyran

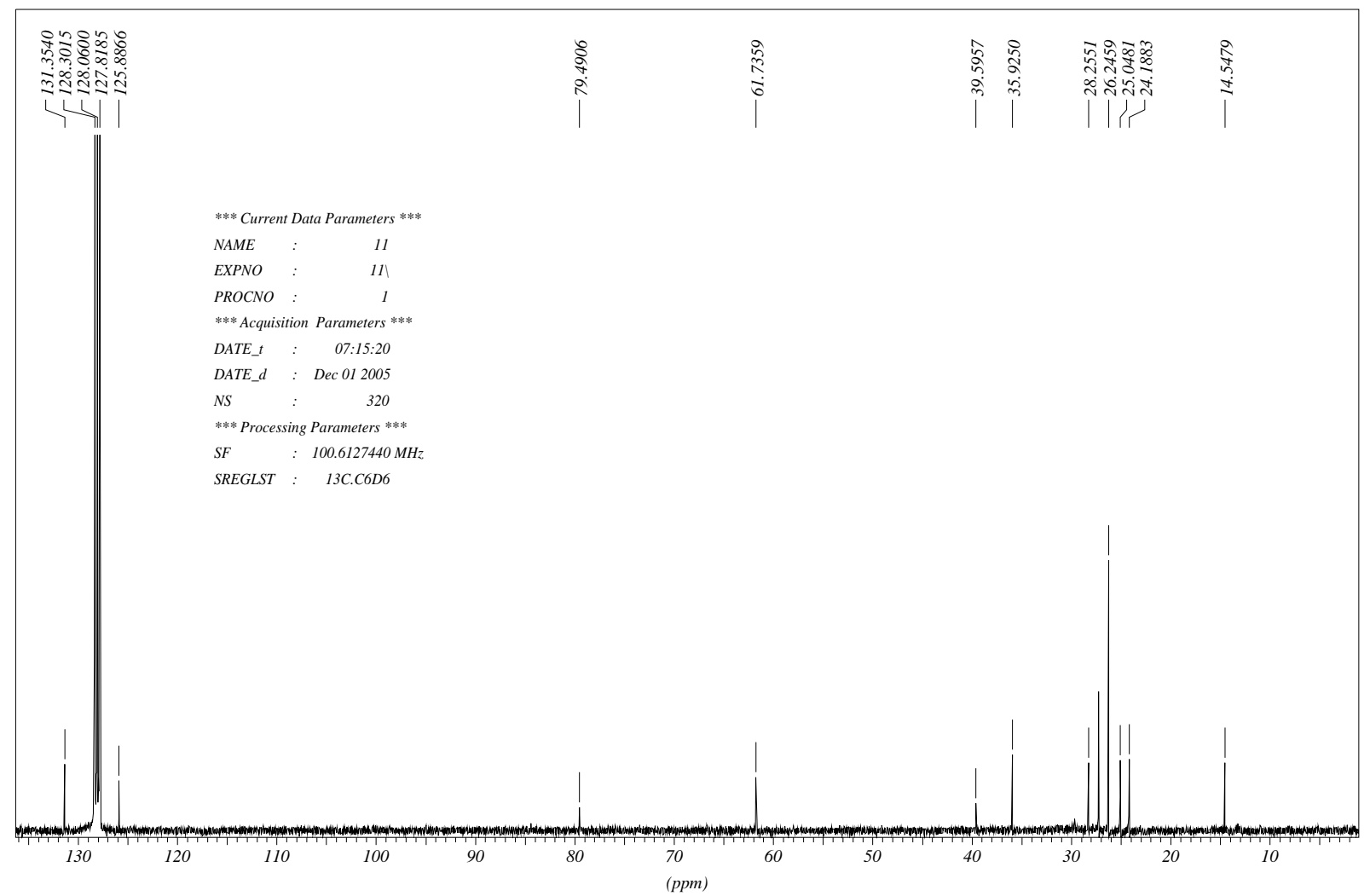


6-Butyl-2,2,6-trimethyl-3,6-dihydro-2H-pyran (8c)

6-Butyl-2,2,6-trimethyl-3,6-dihydro2H-pyran

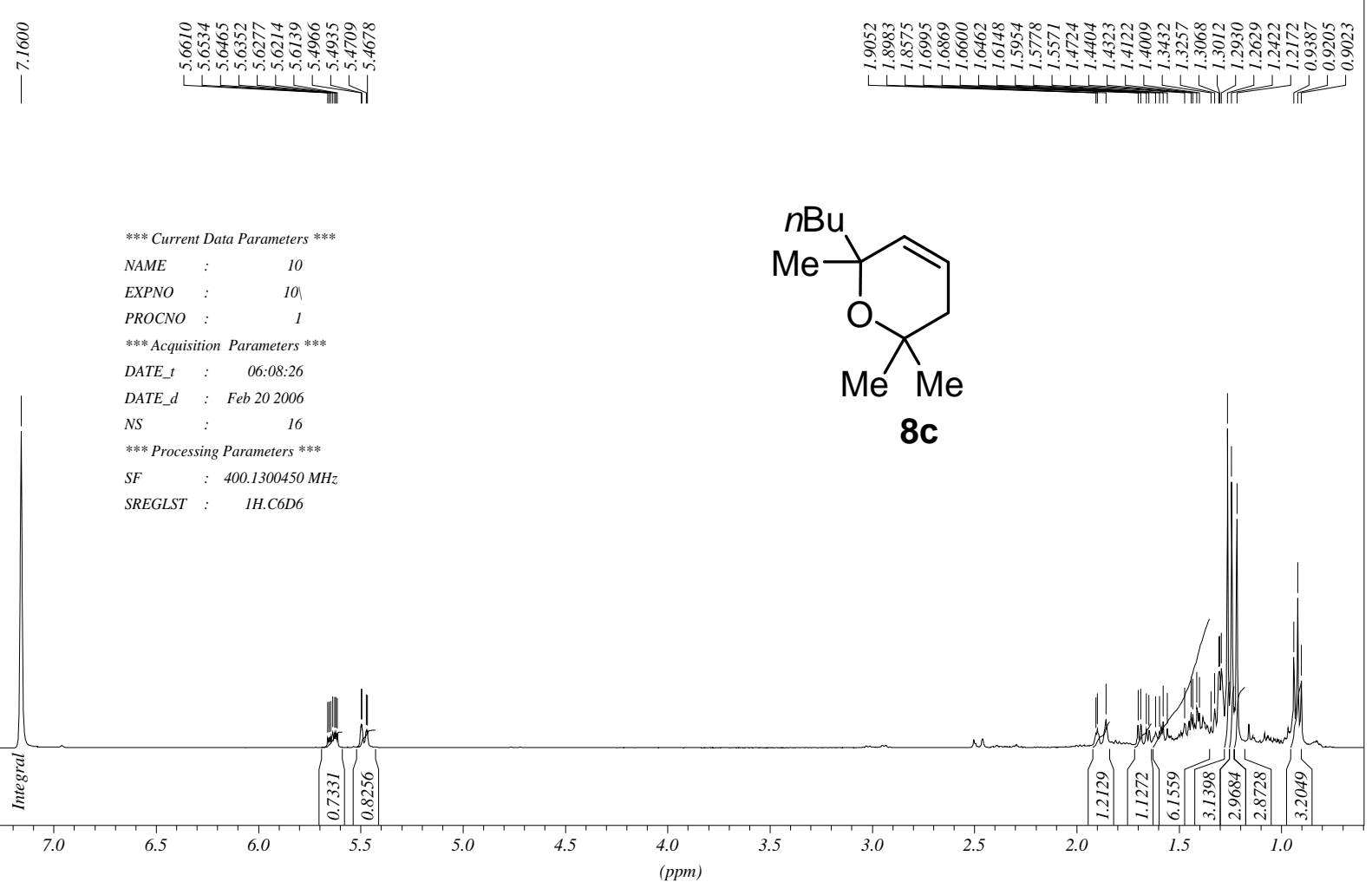

6-Butyl-2,2,6-trimethyl-3,6-dihydro2H-pyran

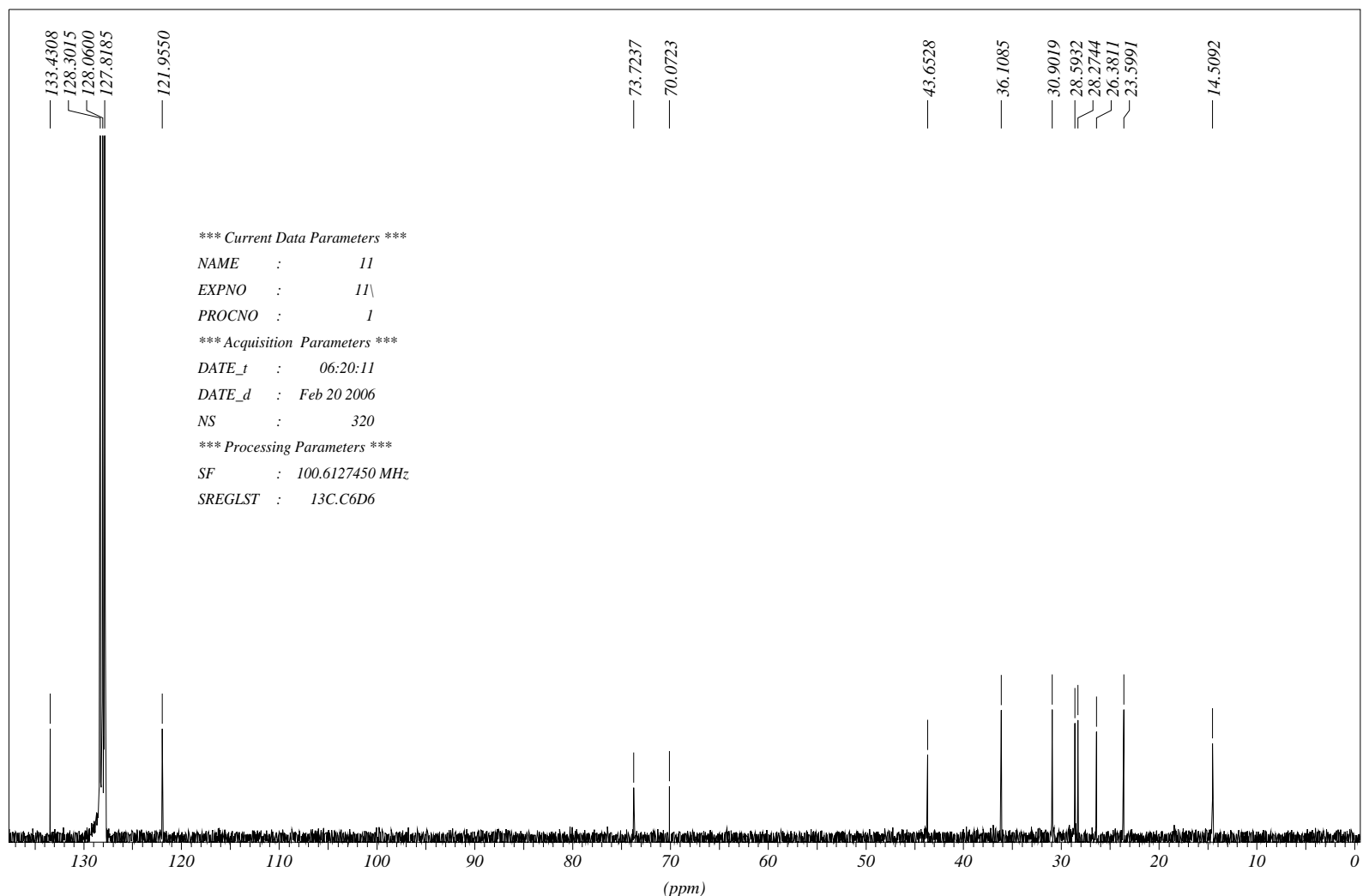


Ethyl 6-butyl-2,2,6-trimethyl-3,6-dihydro-2H-pyran-3-carboxylate (8d)

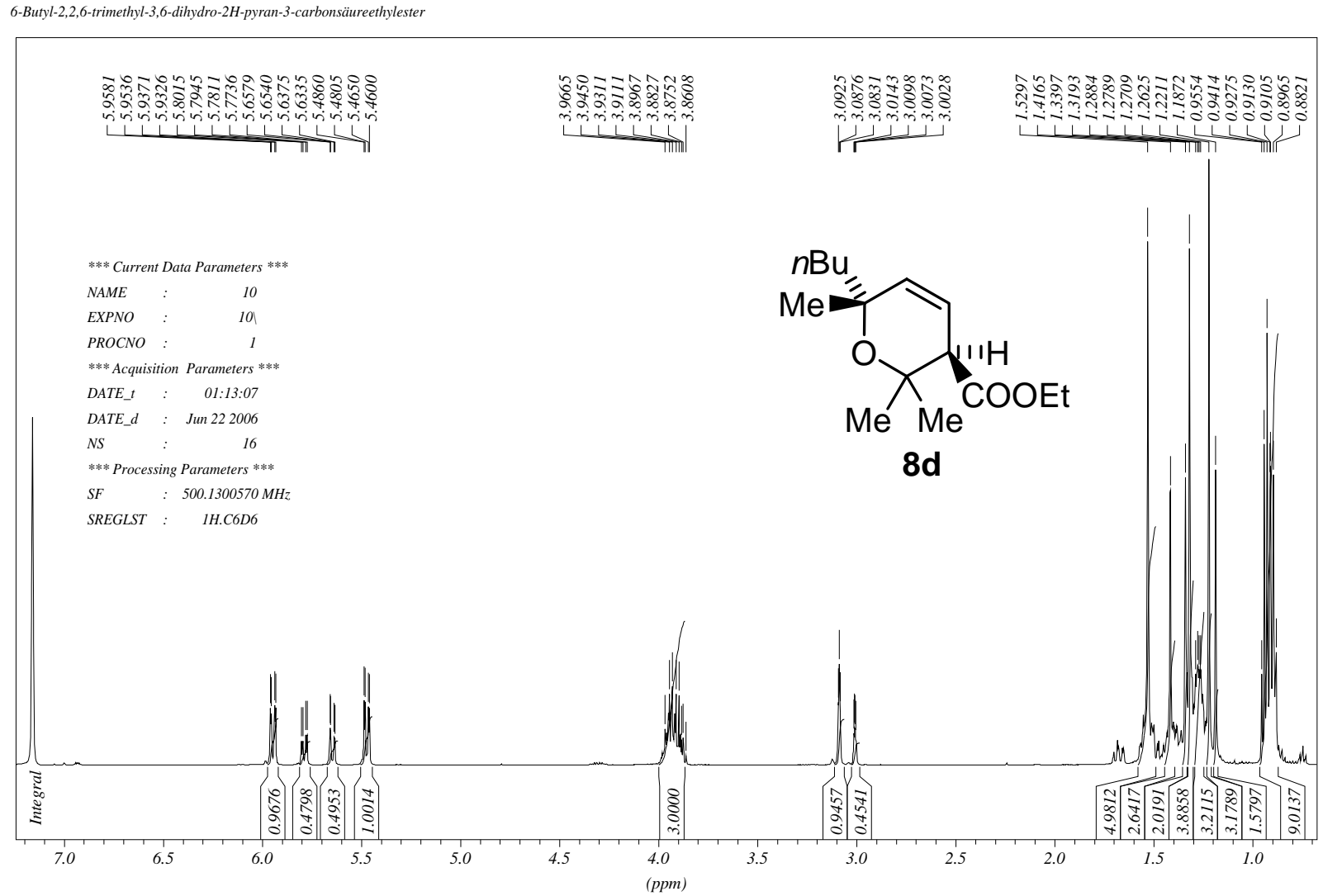

6-Butyl-2,2,6-trimethyl-3,6-dihydro-2H-pyran-3-carbonsäureethylester

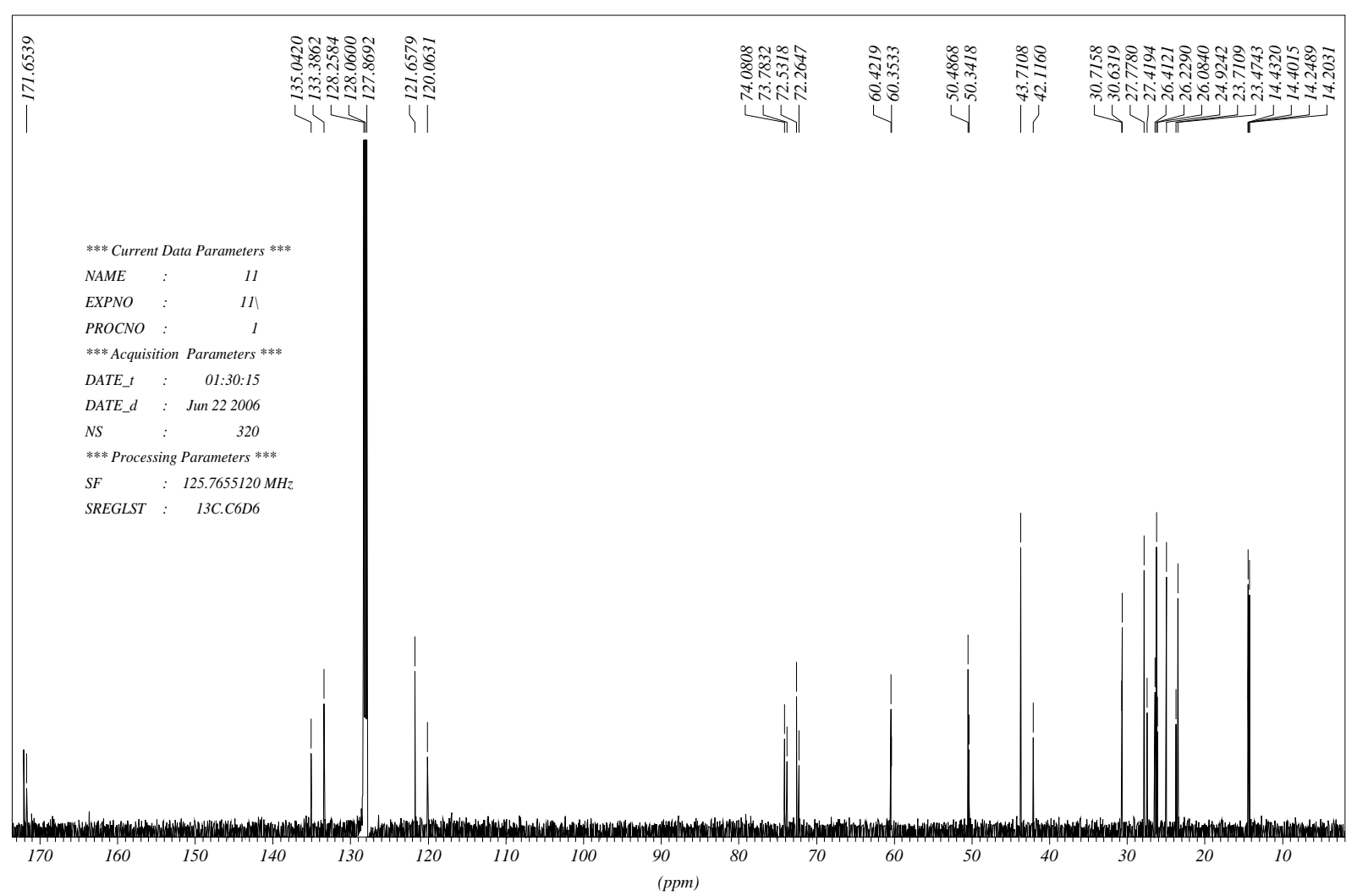


1-Hydroxy-3,6,6-trimethylhepta-3,4-dien-2-yl acetate (9a)

1-Hydroxy-3,6,6-trimethylhepta-3,4-dien-2-yl acetate

i

*** Current Data Parameters ***

NAME : BG21P79C

EXPNO : 10

PROCNO :

****Acquisition Parameters ***

DATE_t : 03:09:03

DATE_d : Dec 062005

NS : $\quad 16$

*** Processing Parameters ***
SF $\quad: \quad 400.1300187 \mathrm{MHz}$

SREGLST : 1 H.C6D6
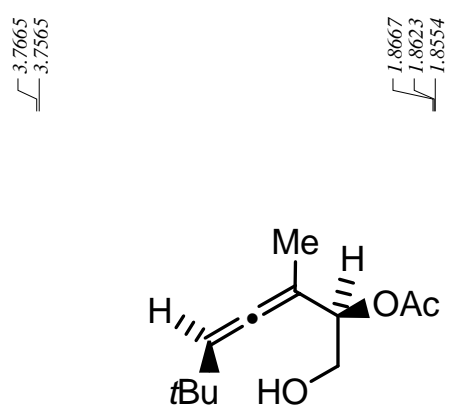

$9 a$
|

$\underset{\substack{2 \\ \infty}}{\infty}$
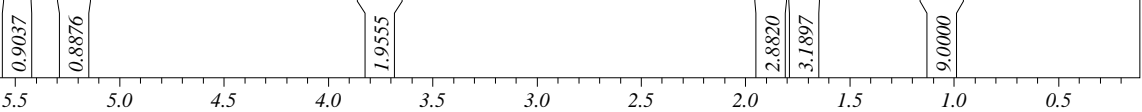

(ppm)

1-Hydroxy-3,6,6-trimethylhepta-3,4-dien-2-yl acetate

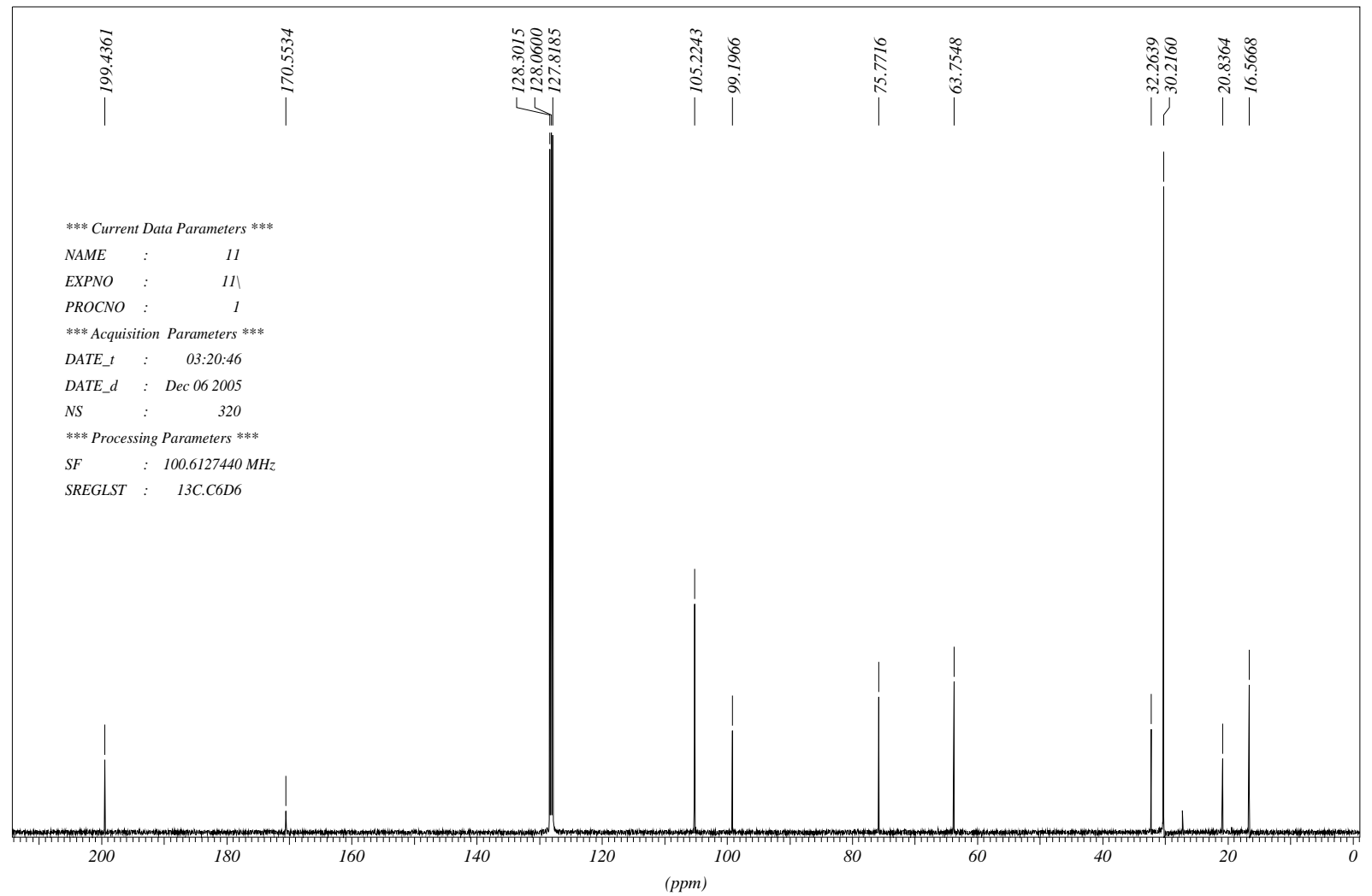


Cis-6-t-Butyl-4-methyl-3,6-dihydro-2H-pyran-3-yl acetate (10a)

6-tert-Butyl-4-methyl-3,6-dihydro-2H-pyran-3-ylacetat

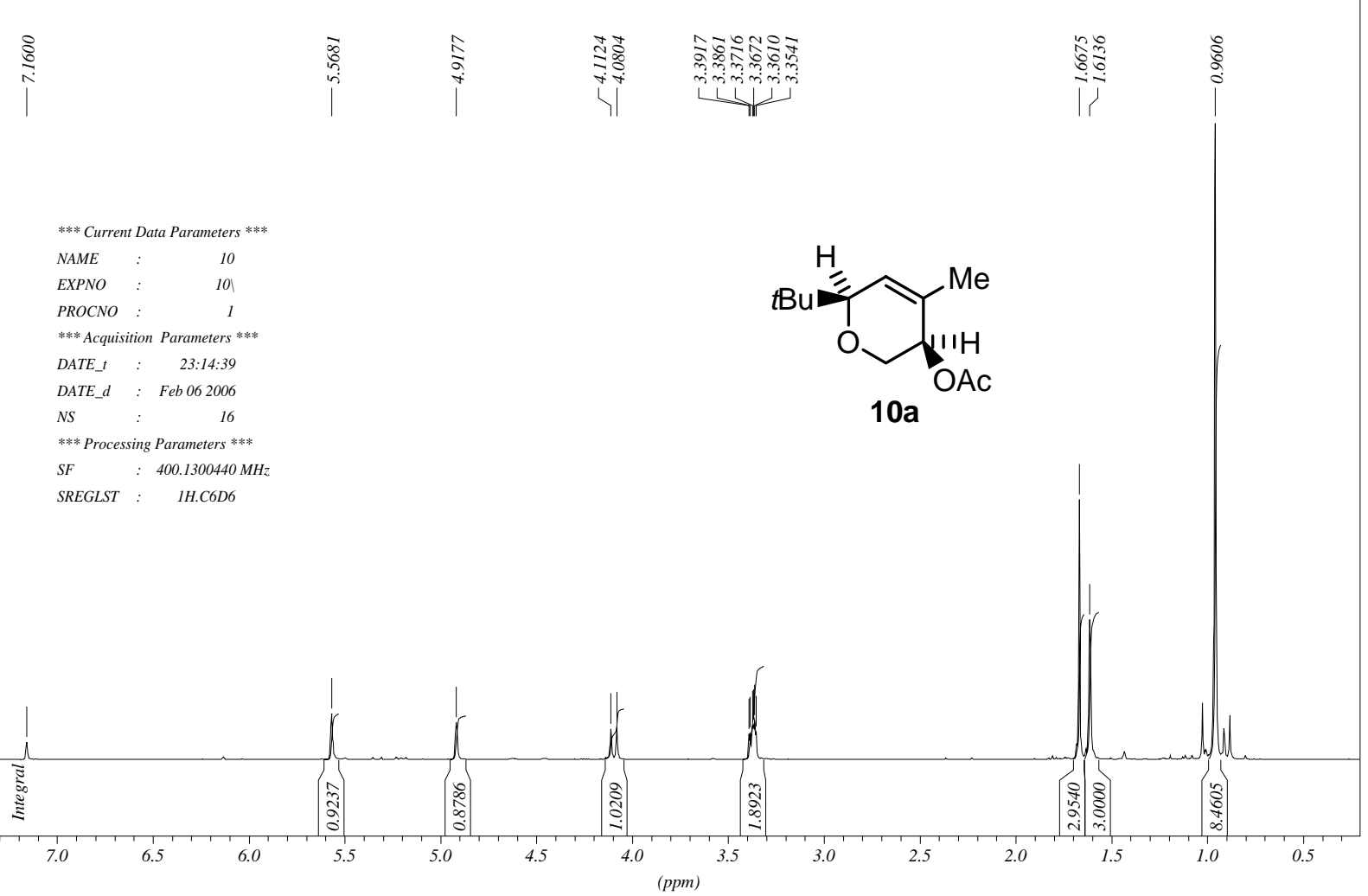

6-tert-Butyl-4-methyl-3,6-dihydro-2H-pyran-3-ylacetat

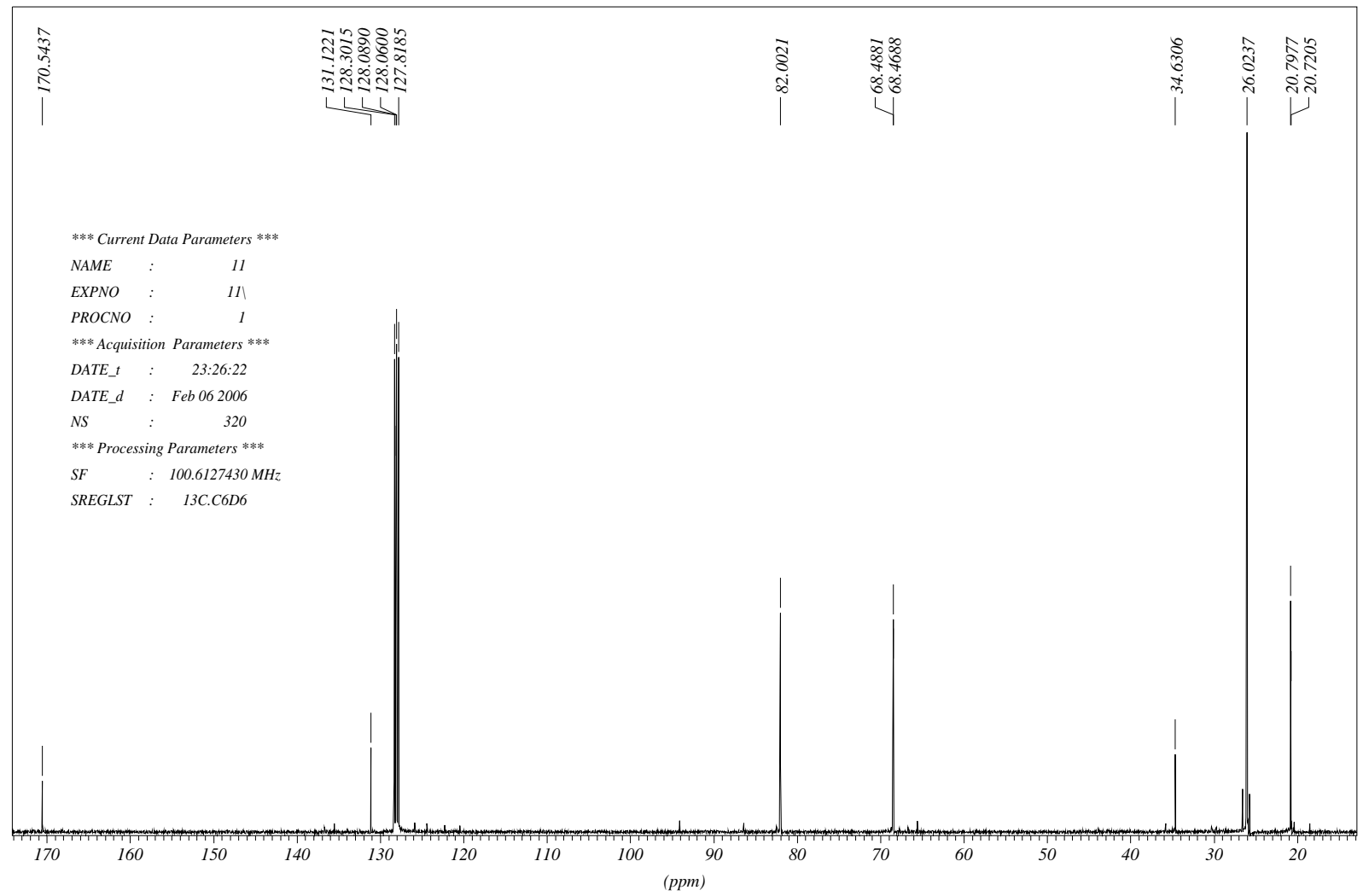


Trans-2-Acetoxymethyl-5-t-butyl-3,5-dimethyl-2,5-dihydrofuran (11a)

5-tert-Butyl-2-acetoxymethyl-3,5-dimethyl-2,5-dihydrofuran

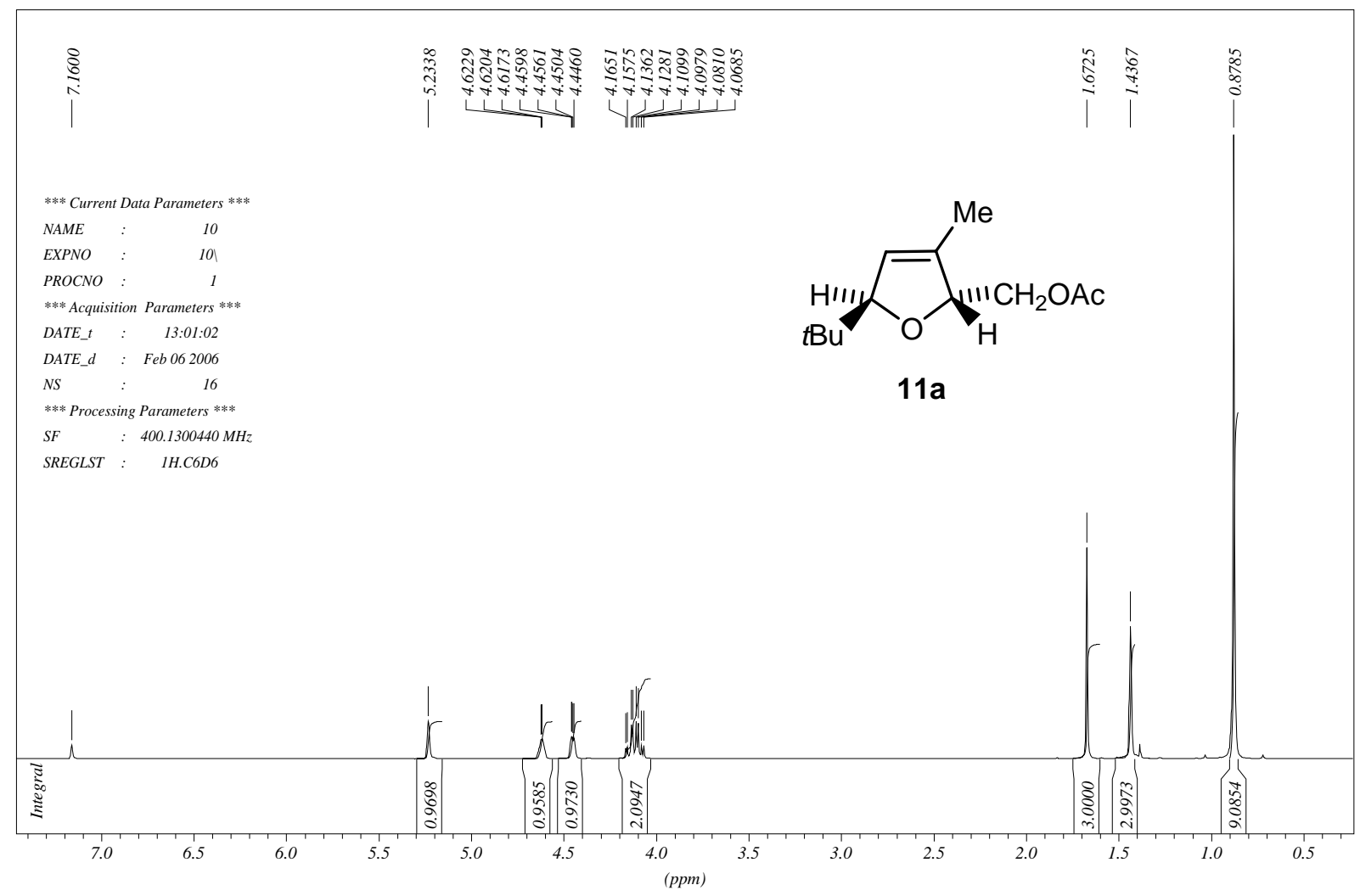

5-tert-Butyl-2-acetoxymethyl-3,5-dimethyl-2,5-dihydrofuran

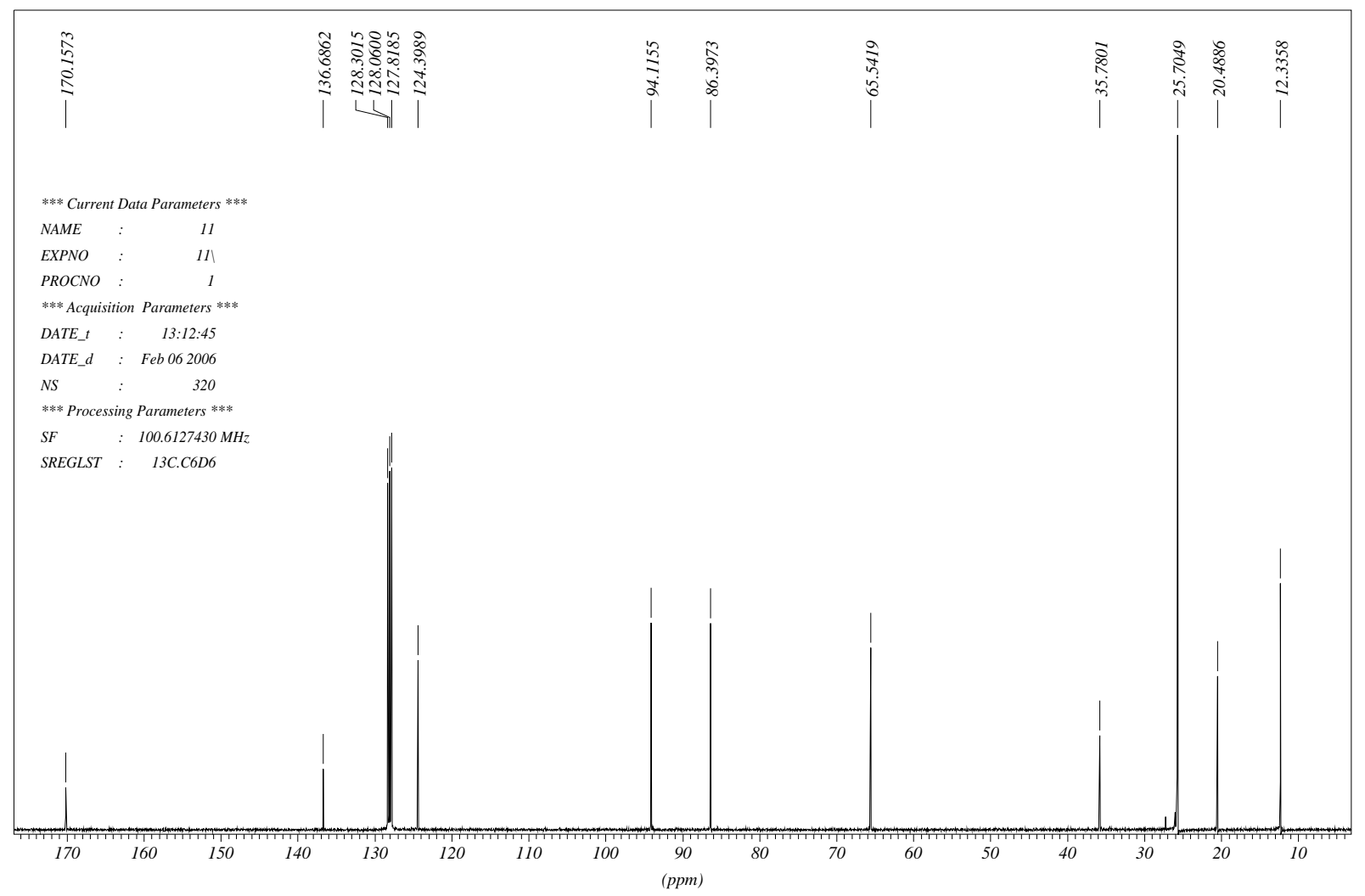


Trans-6-t-Butyl-4-methyl-3,6-dihydro-2H-pyran-3-yl acetate (10b)

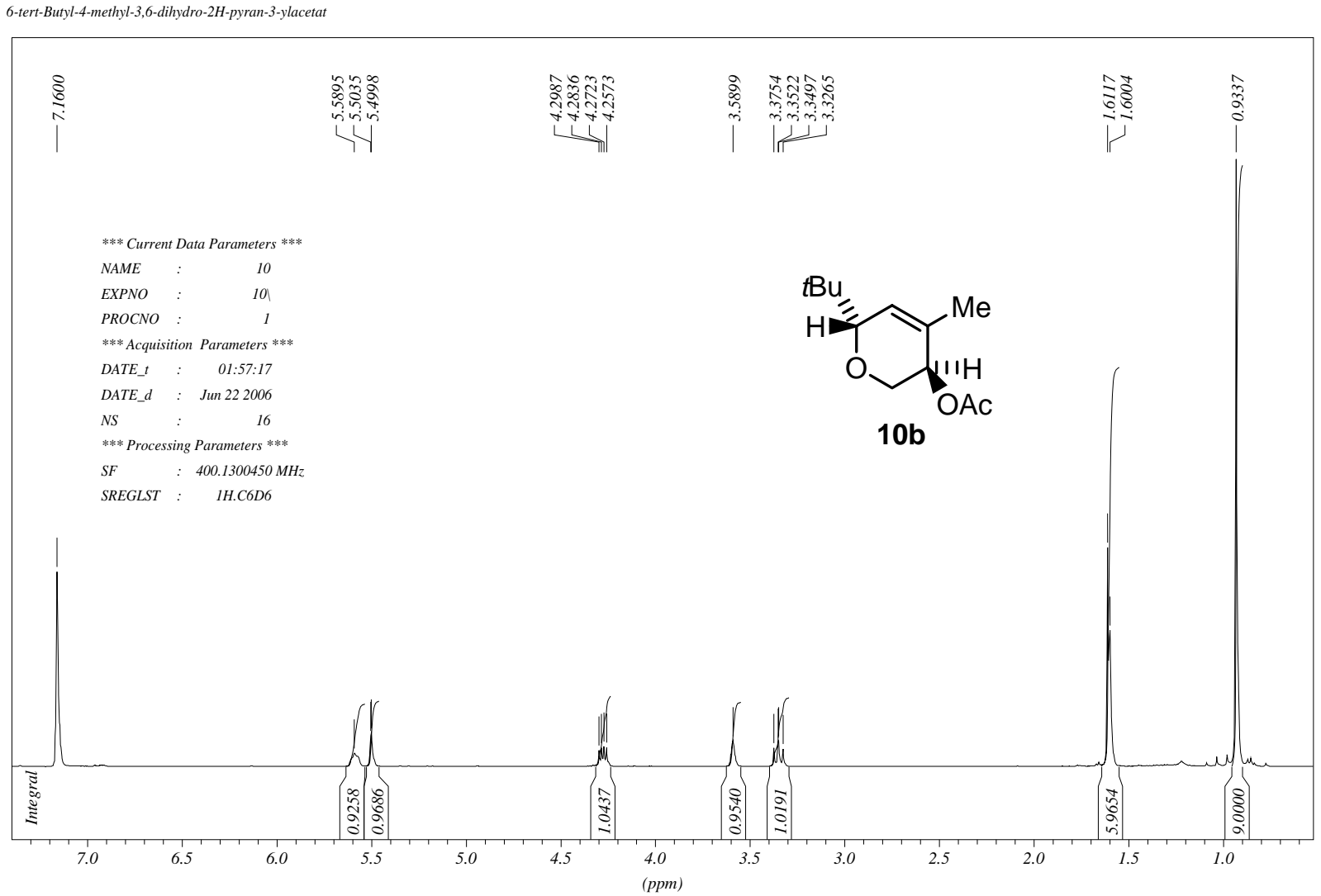

6-tert-Butyl-4-methyl-3,6-dihydro-2H-pyran-3-ylacetat

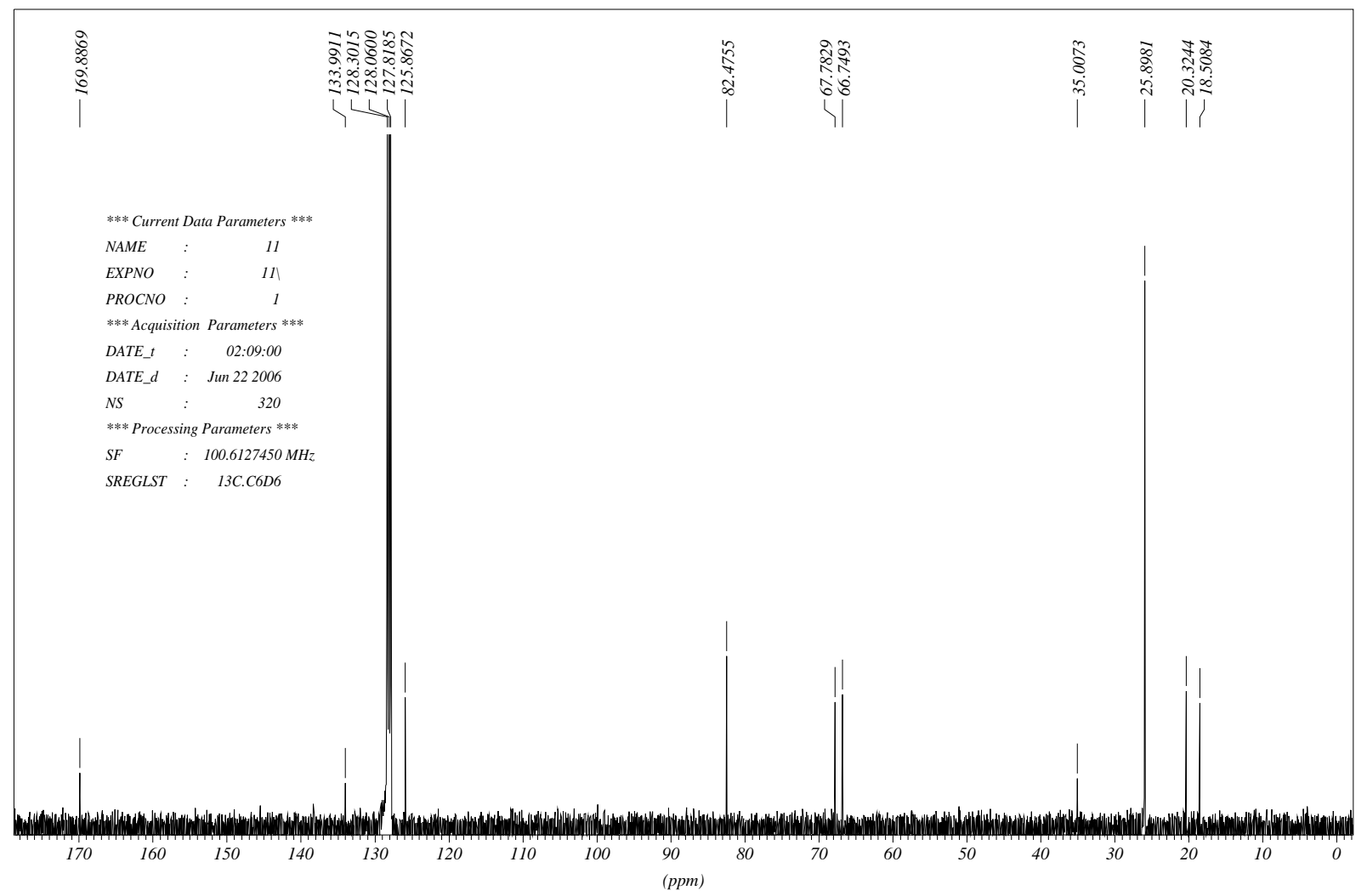


Cis-2-Acetoxymethyl-5-t-butyl-3,5-dimethyl-2,5-dihydrofuran (11b)

5-tert-Butyl-2-acetoxymethyl-3,5-dimethyl-2,5-dihydrofuran

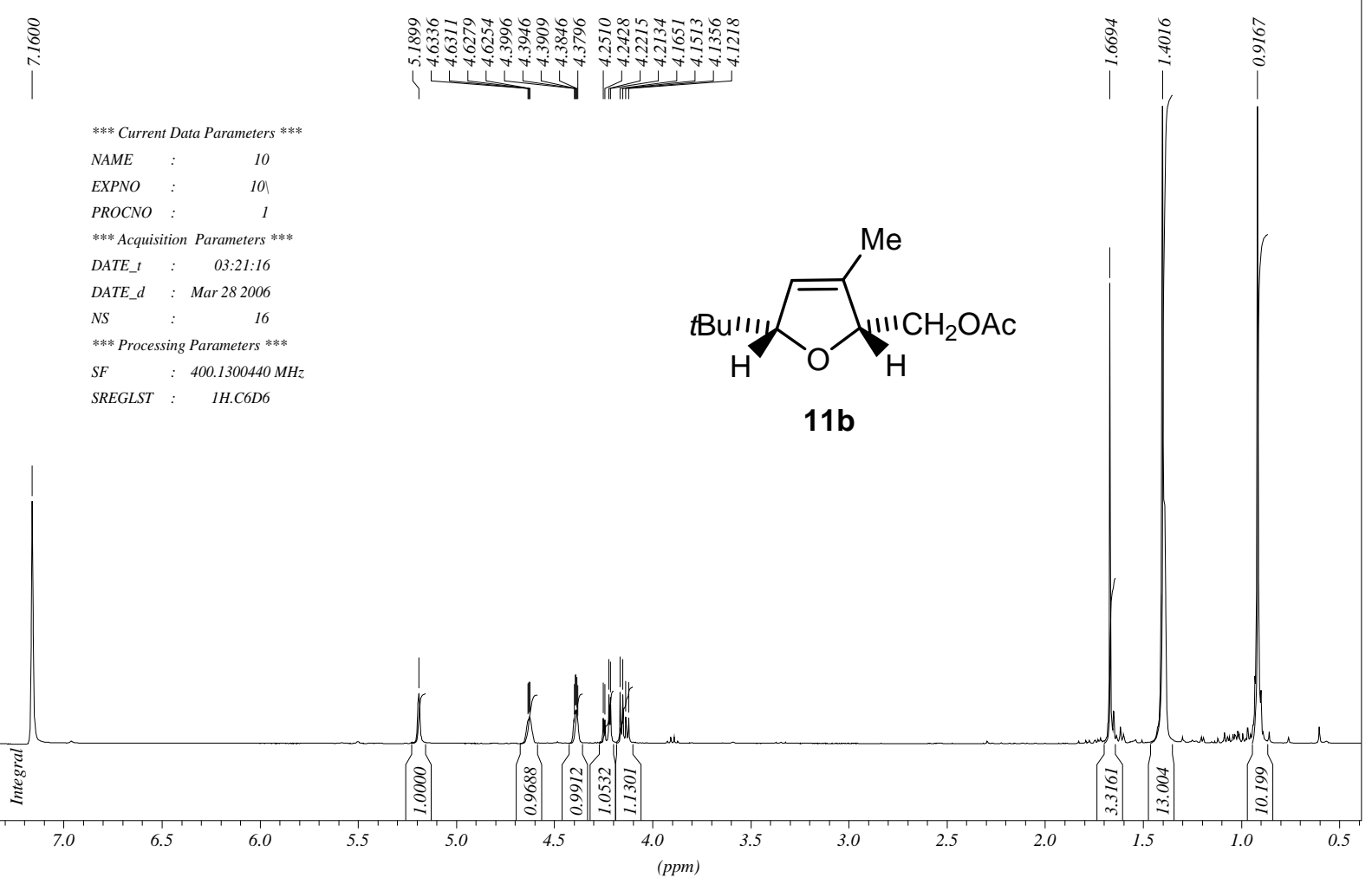

5-tert-Butyl-2-acetoxymethyl-3,5-dimethyl-2,5-dihydrofuran

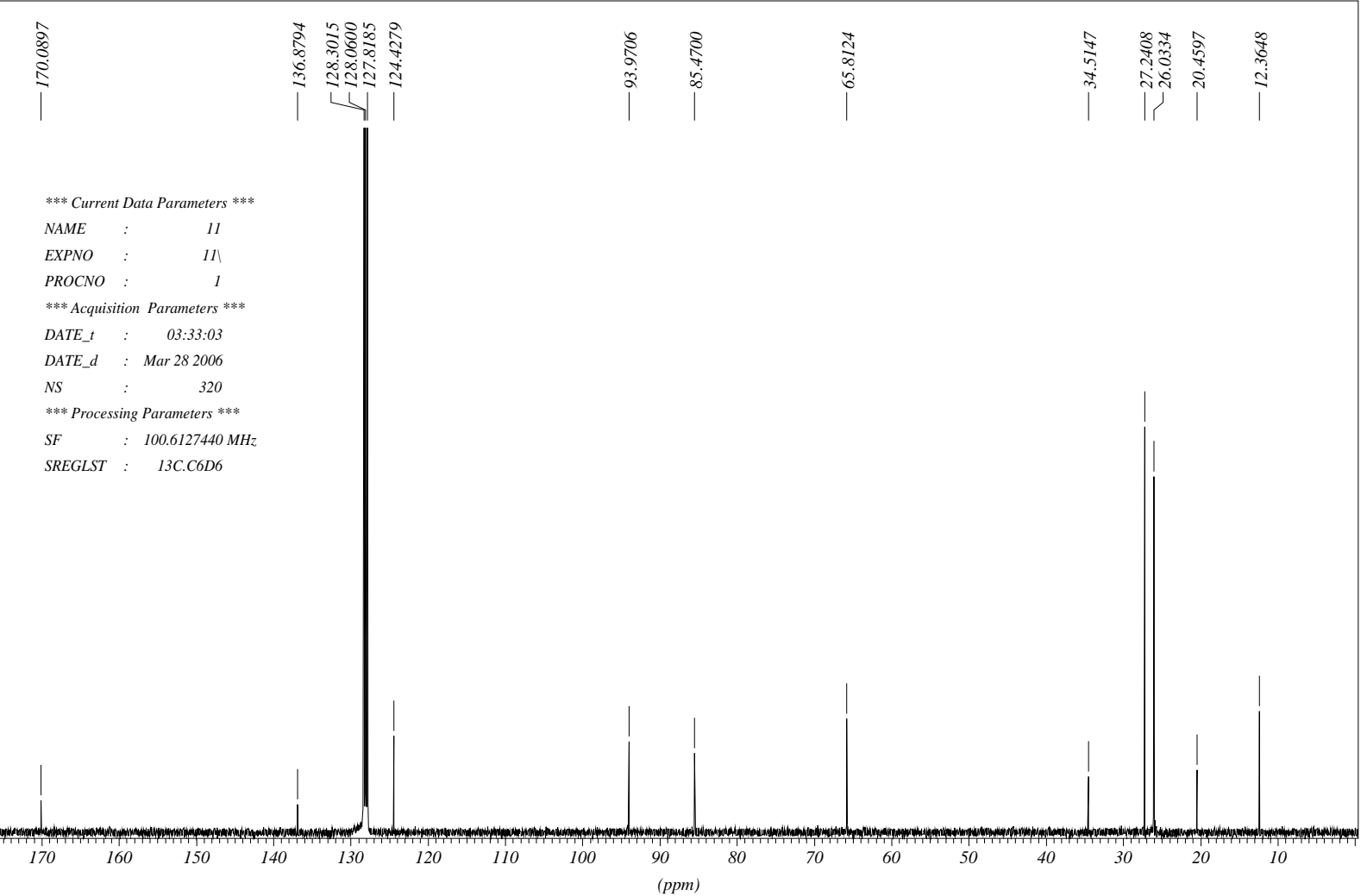


Trans-6-Butyl-4-methyl-3,6-dihydro-2H-pyran-3-yl acetate (10c)
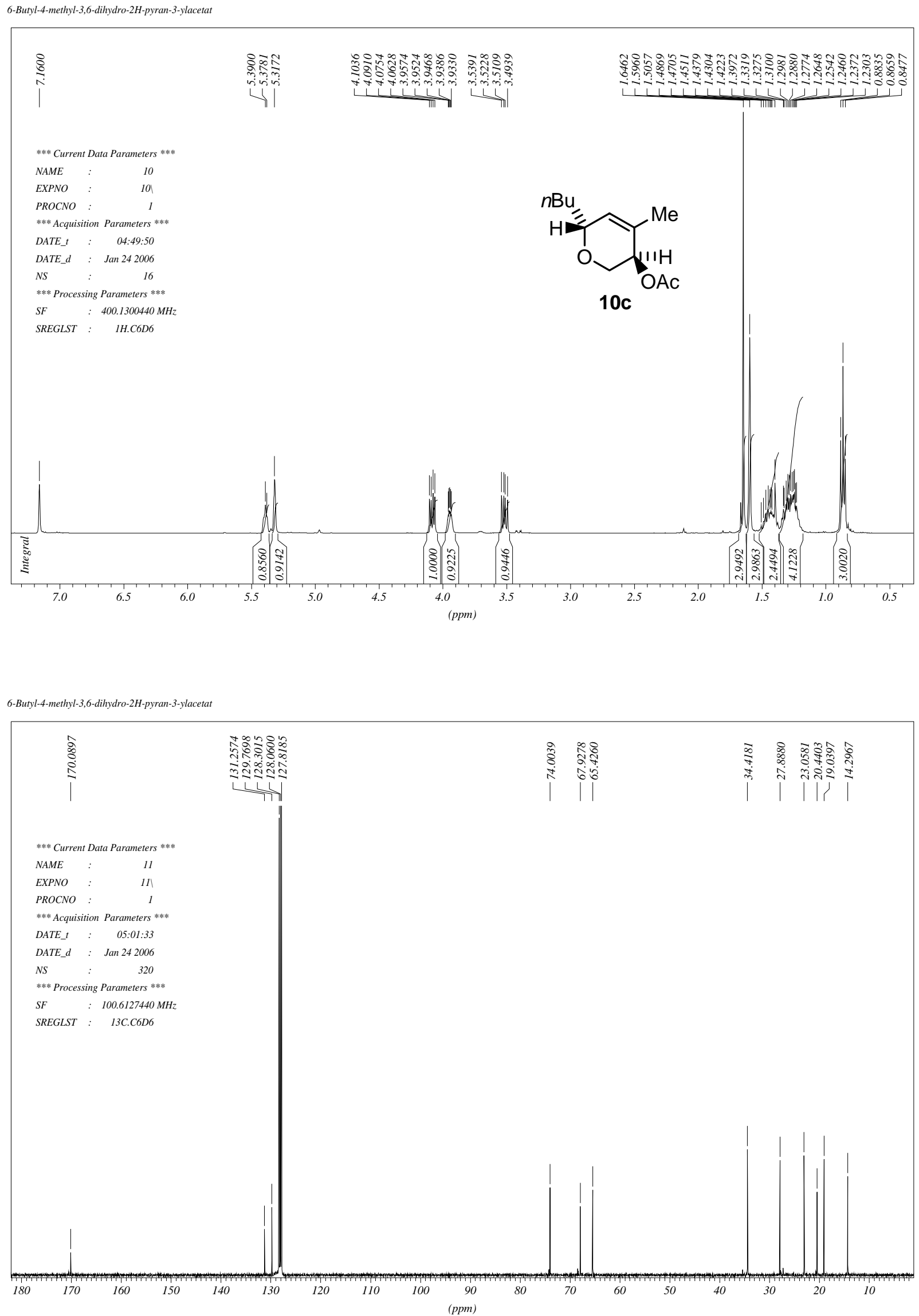
Trans-2-Butyl-5-methoxy-5,6-dihydro-2H-pyran (10d)

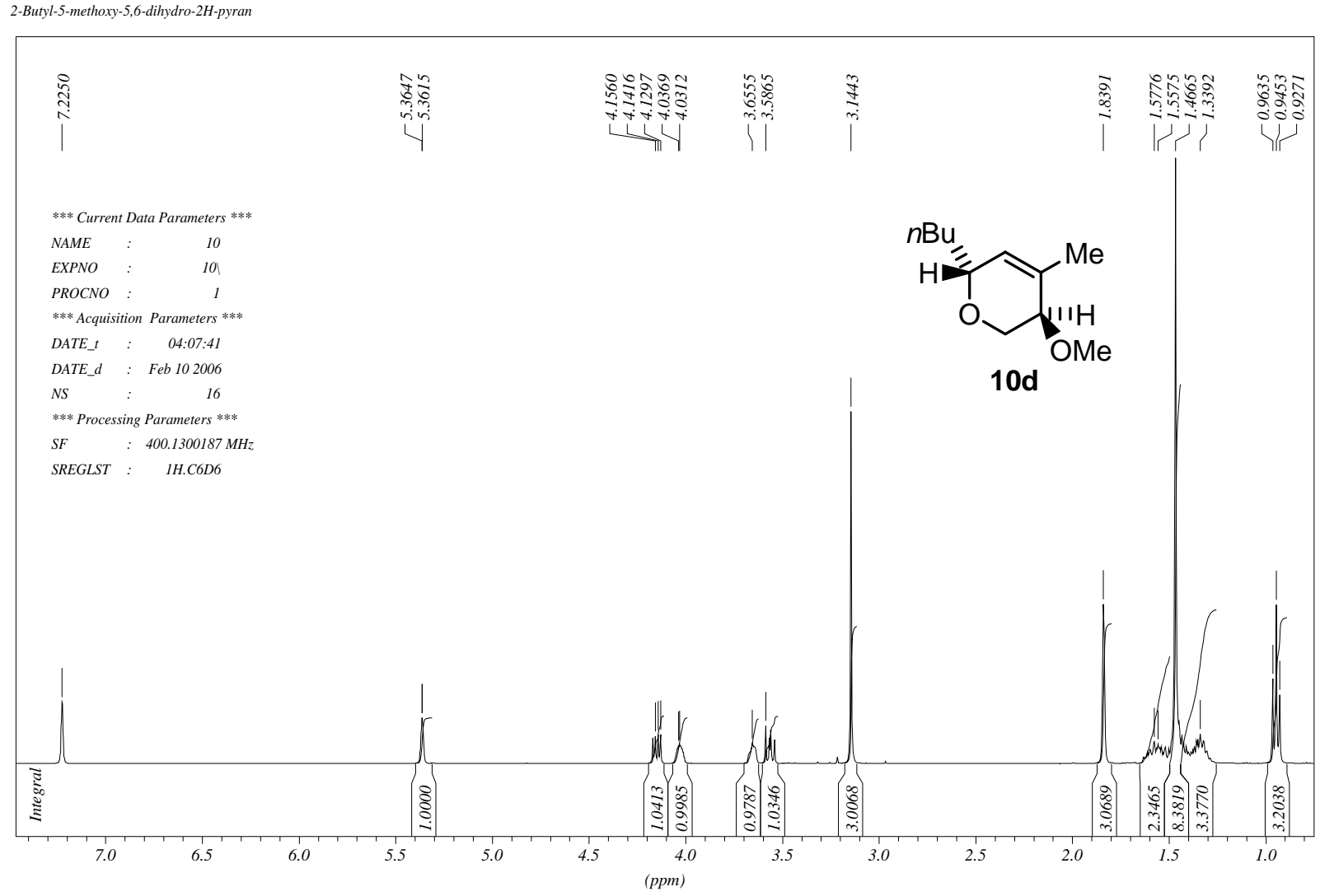

2-Butyl-5-methoxy-5,6-dihydro-2H-pyran

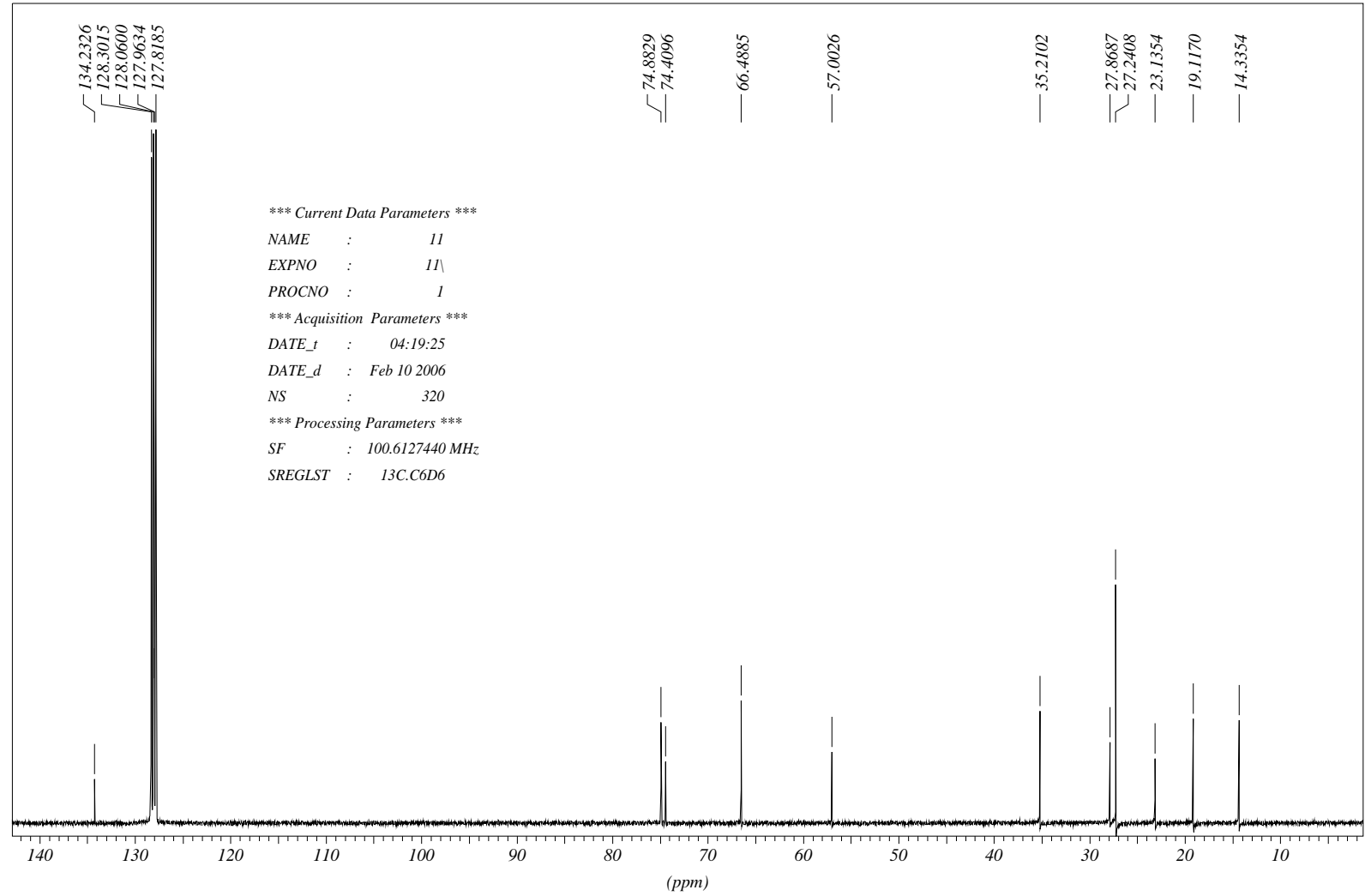


Trans-4-Methyl-6-phenyl-3,6-dihydro-2H-pyran-3-yl acetate (10e)

4-Methyl-6-phenyl-3,6-dihydro-2H-pyran-3-ylacetat

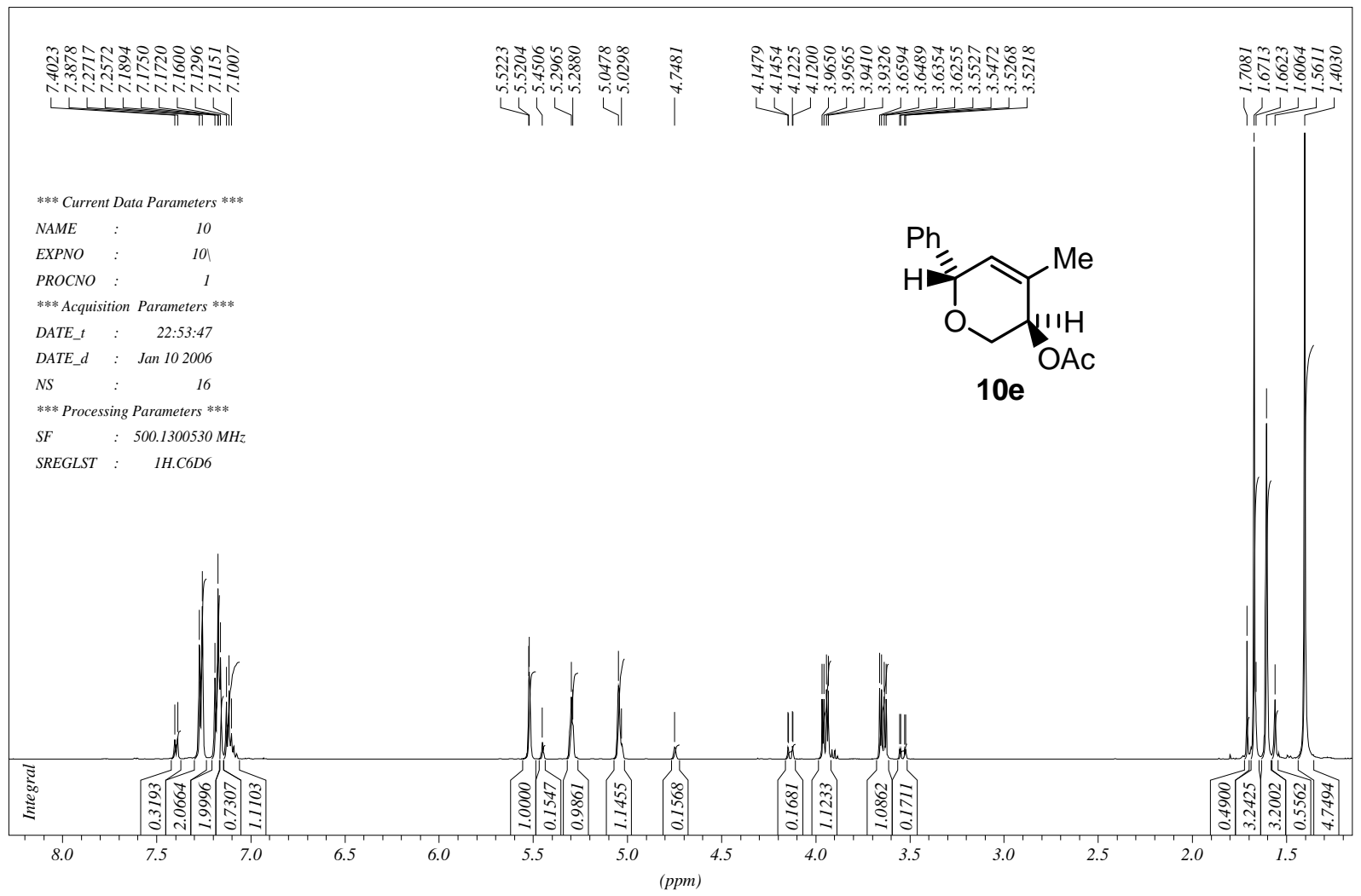

4-Methyl-6-phenyl-3,6-dihydro-2H-pyran-3-ylacetat

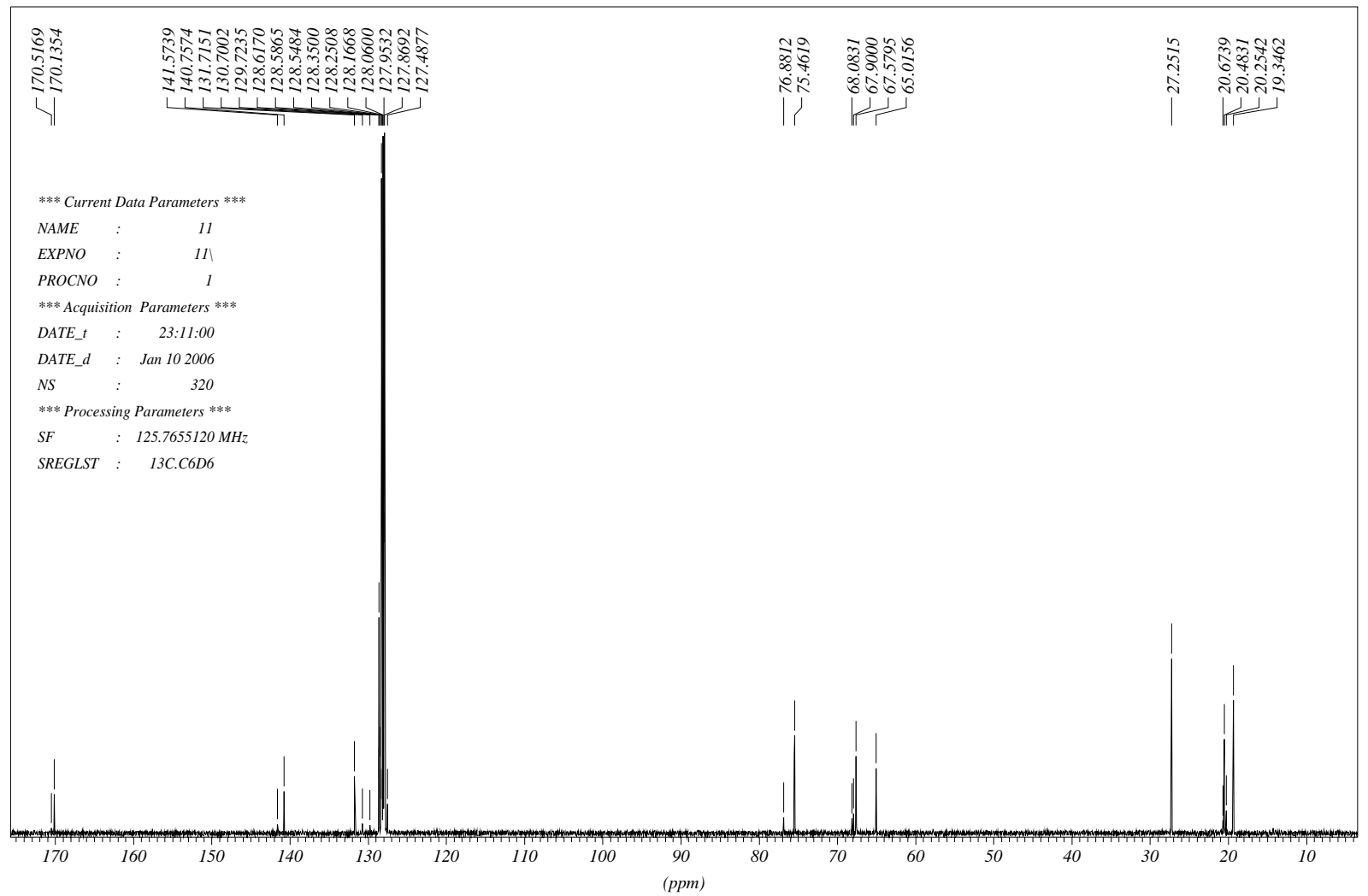


Trans-5-Methoxy-4-methyl-2-phenyl-5,6-dihydro-2H-pyran (10f)

5-Methoxy-4-methyl-2-phenyl-5,6-dihydro-2H-pyran

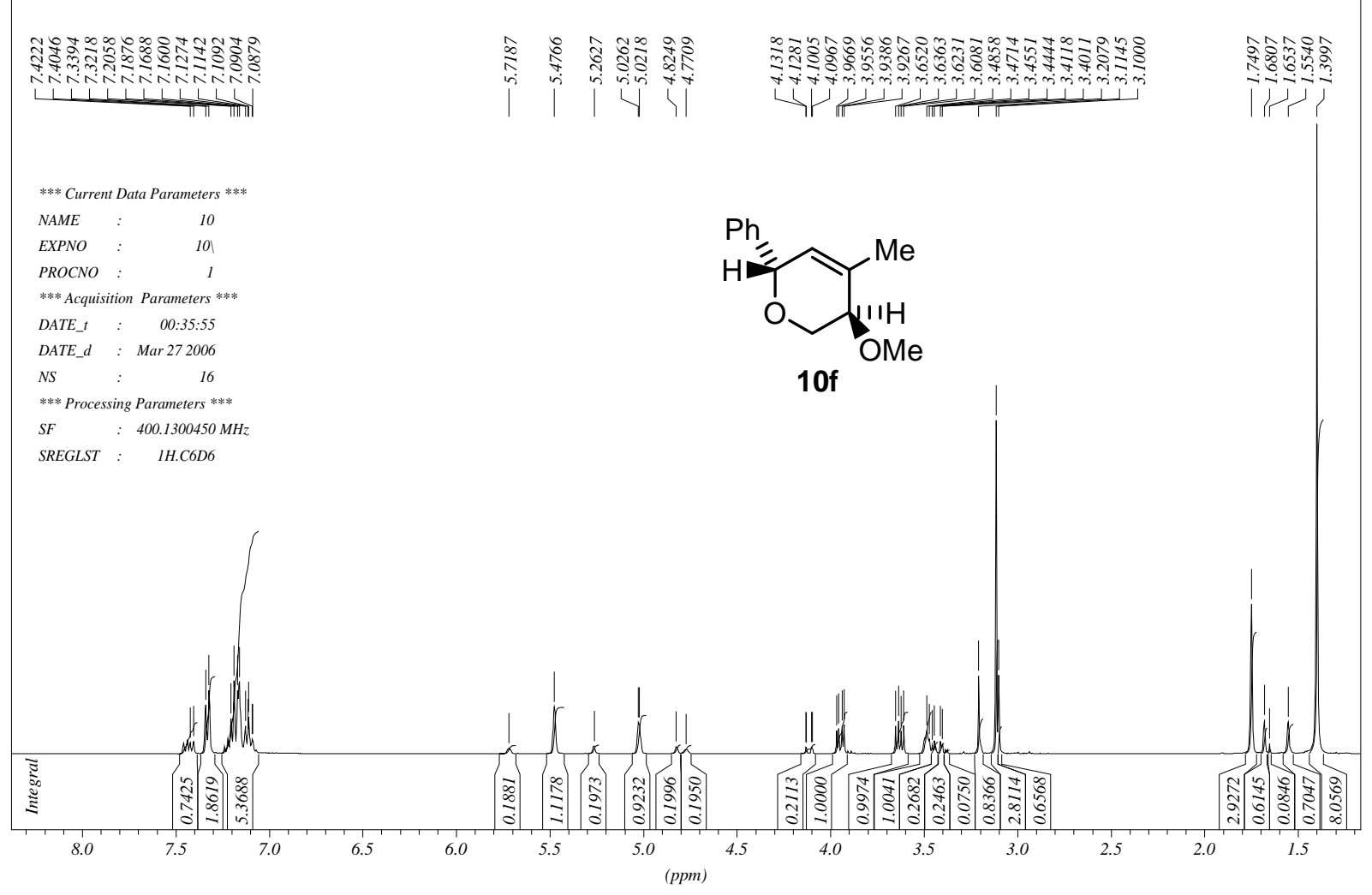

5-Methoxy-4-methyl-2-phenyl-5,6-dihydro-2H-pyran

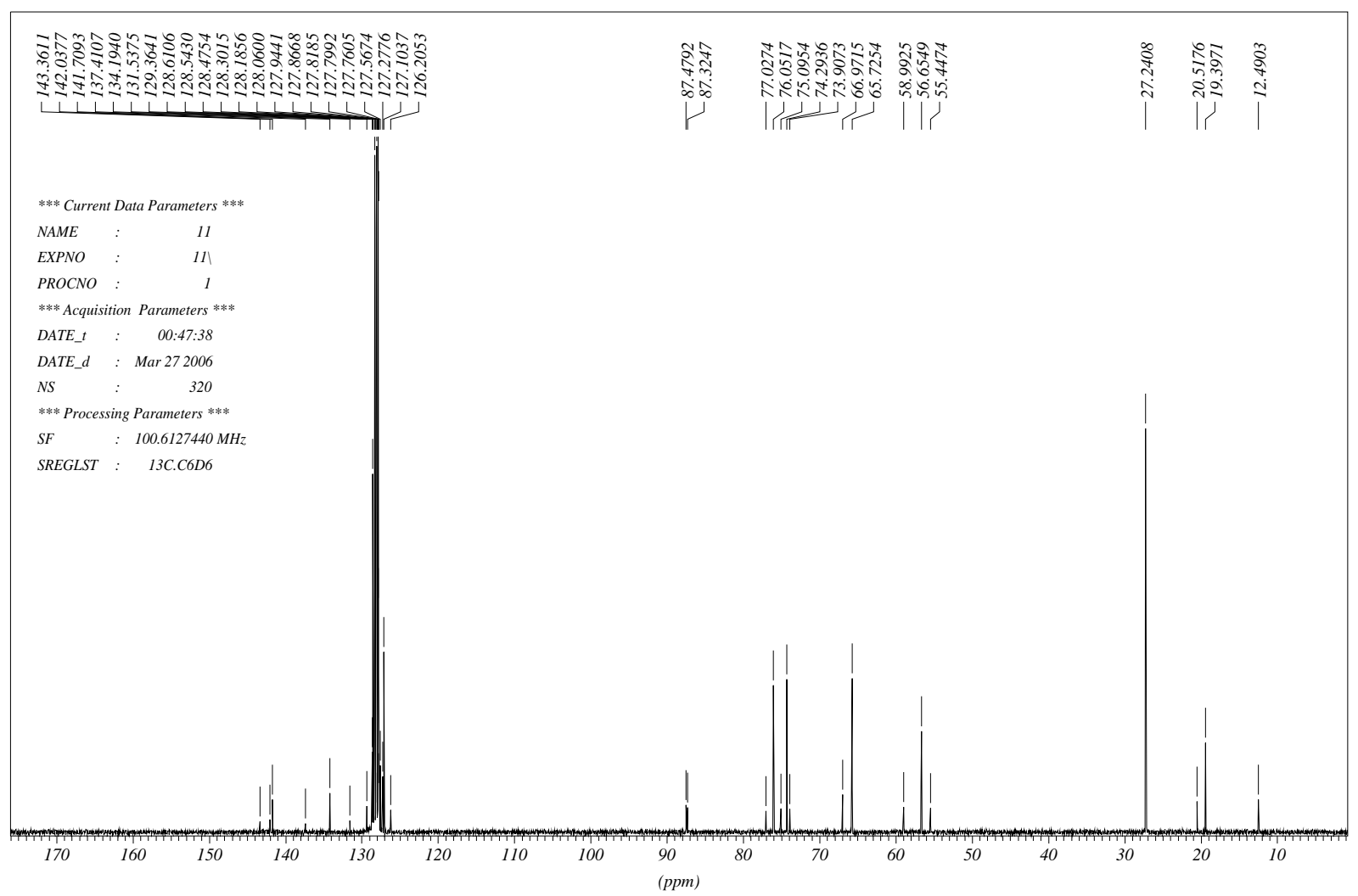


2-Butyl-2-methyl-1,2,5,6-tetrahydropyridin (13)

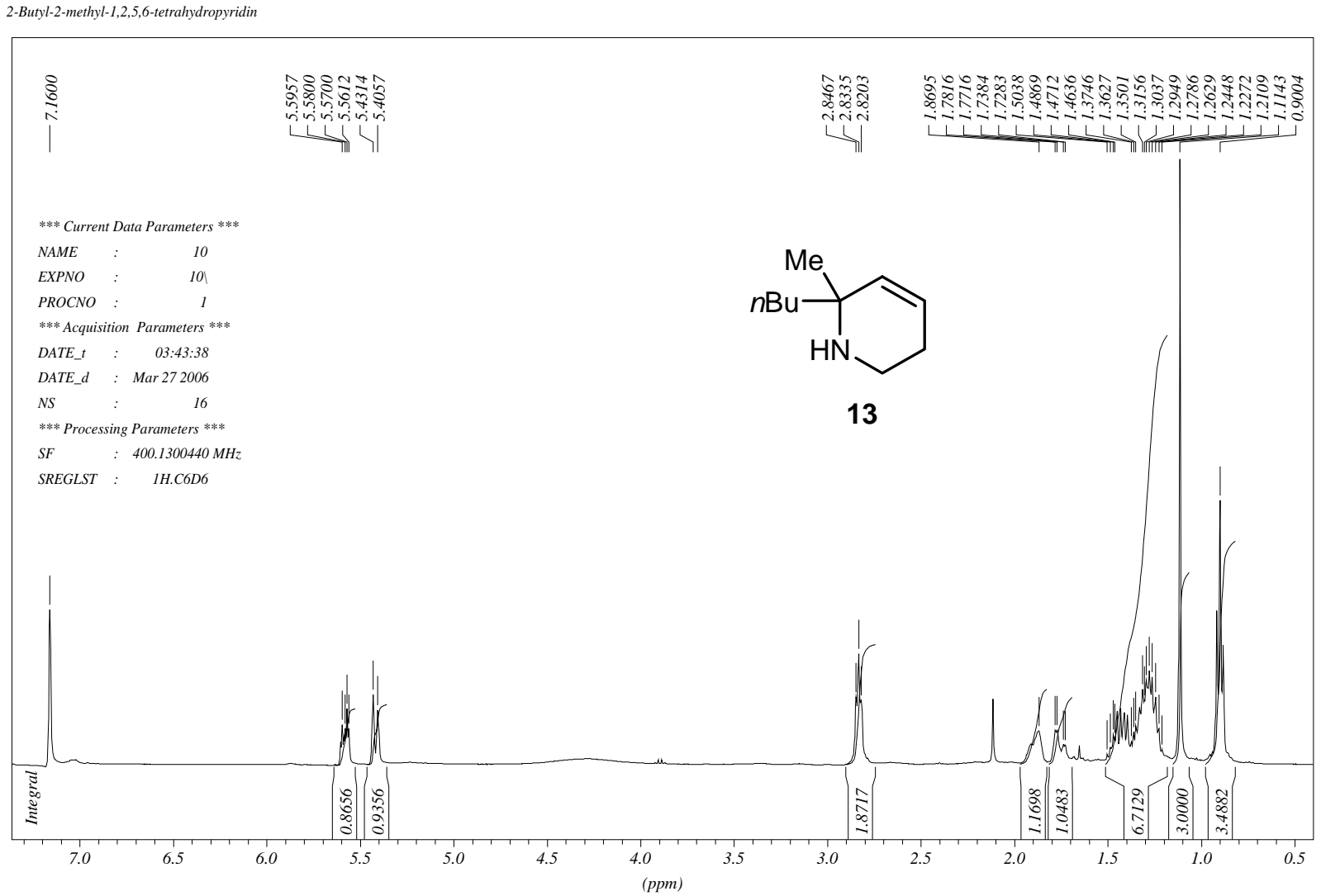

2-Butyl-2-methyl-1,2,5,6-tetrahydropyridin

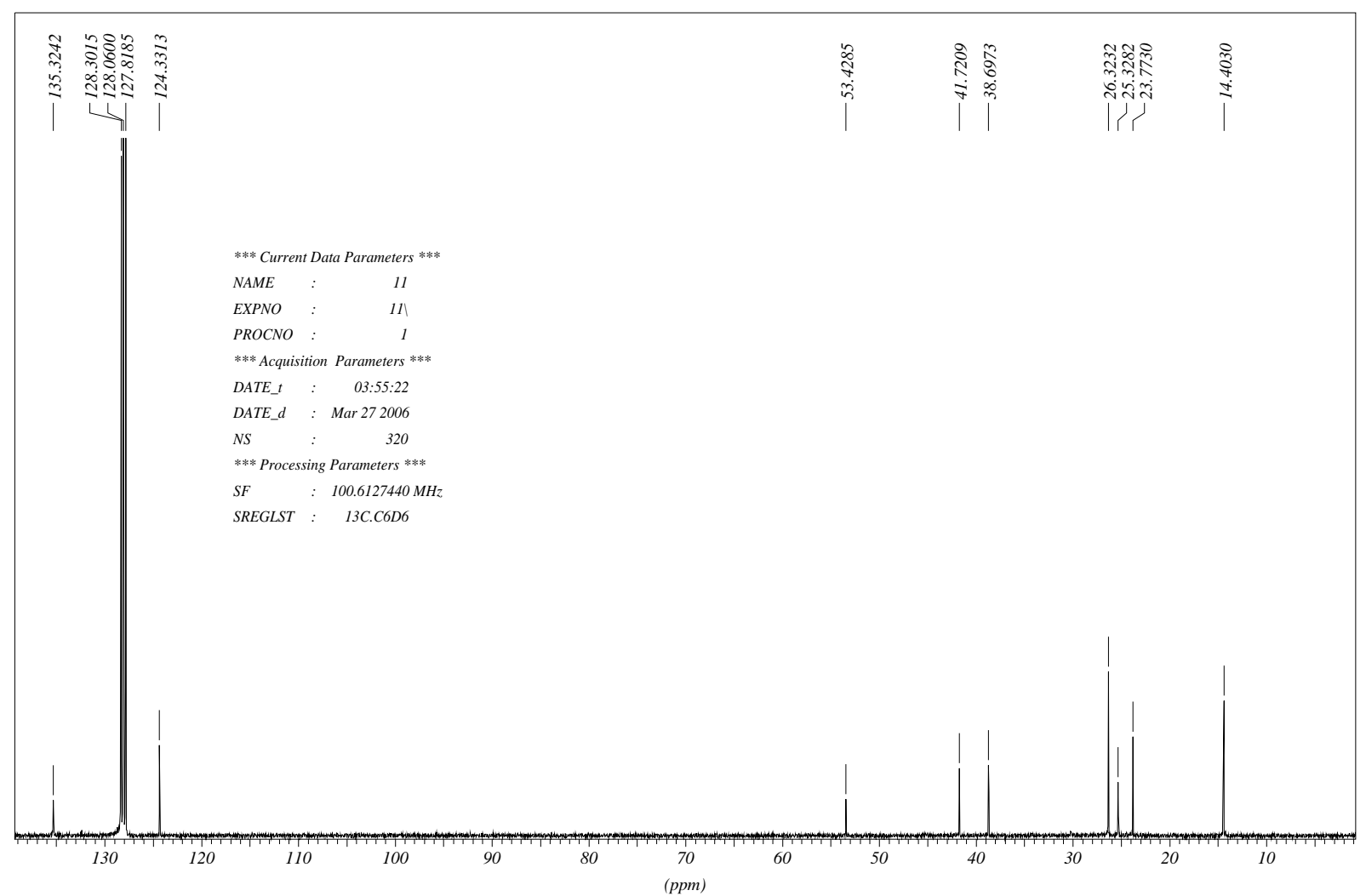

